\title{
TLR9 regulates the mycobacteria-elicited pulmonary granulomatous immune response in mice through DC-derived Notch ligand delta-like 4
}

Toshihiro Ito, ${ }^{1,2}$ Matthew Schaller, ${ }^{1}$ Cory M. Hogaboam, ${ }^{1}$ Theodore J. Standiford, ${ }^{3}$ Matyas Sandor ${ }^{4}$ Nicholas W. Lukacs, ${ }^{1}$ Stephen W. Chensue, ${ }^{1,5}$ and Steven L. Kunkel ${ }^{1}$

\author{
1Department of Pathology, University of Michigan Medical School, Ann Arbor, Michigan, USA. 2Second Department of Internal Medicine, \\ Nara Medical University, Kashihara, Nara, Japan. ${ }^{3}$ Division of Pulmonary and Critical Care Medicine, Department of Medicine, \\ University of Michigan Medical Center, Ann Arbor, Michigan, USA. ${ }^{4}$ Department of Pathology and Laboratory Medicine, \\ University of Wisconsin School of Medicine and Public Health, Madison, Wisconsin, USA. ${ }^{5}$ Department of Pathology and Laboratory Medicine, \\ Veterans Affairs Ann Arbor Healthcare System, Ann Arbor, Michigan, USA.
}

\begin{abstract}
TLR9 activation is important for the maintenance of mycobacteria-elicited pulmonary granulomatous responses, hallmarks of protective immune responses following mycobacterial infection. However, the mechanism or mechanisms underlying this effect of TLR9 are not clear. Here, we show that Tlr9-deficient mice challenged with a Mycobacterium antigen display an altered Th17 cytokine profile, decreased accumulation of granulomaassociated myeloid DCs, and profoundly impaired delta-like 4 (dll4) Notch ligand expression. Mechanistic analysis revealed that WT bone marrow-derived DCs but not macrophages promoted the differentiation of Th17 cells from bacillus Calmette-Guérin-challenged (BCG-challenged) lung CD4 ${ }^{+} \mathrm{T}$ cells. Both lung and bone marrow DCs isolated from Tlr9-deficient mice inoculated with Mycobacterium antigen expressed lower levels of dll4 Notch ligand than the same cells isolated from WT mice. Passively immunizing WT mice with neutralizing antibodies specific for d114 during granuloma formation resulted in larger granulomas and lower levels of Th17-related cytokines. In addition, dll4 specifically regulated Th17 activation in vitro. Together, these results suggest d114 plays an important role in promoting Th17 effector activity during a mycobacterial challenge. Furthermore, TLR9 seems to be required for optimal dll4 expression and the regulation of Mycobacterium antigen-elicited granuloma formation in mice.
\end{abstract}

\section{Introduction}

Granulomas represent a spectrum of inflammatory sequestration responses that may be initiated by a variety of agents, including noninfectious environmental factors and infectious microbial pathogens $(1,2)$. One of the most well-characterized bacteriainduced granulomagenic diseases is tuberculosis. It has been estimated that one-third of the world's population is infected with Mycobacterium tuberculosis - resulting in more than 8 million cases of active tuberculosis with 1.6 million deaths globally in 2005 alone (3). While the mechanism or mechanisms of granuloma formation are not clear, this distinct cellular response is considered a histologic hallmark for a protective immune response. The granulomatous reaction is likely initiated by innate immune events; however, the continued intensity and chronicity is dependent upon the engagement of acquired immune mechanisms. We previously described a model of polarized Th1 cell-mediated anamnestic pulmonary granulomatous inflammation in sensitized mice induced by pulmonary embolization of agarose beads coated with covalently bound Ags of mycobacteria-derived purified protein derivative (PPD) (4). Cytokine and chemokine analyses of this model indicated protein profiles consistent with a predominant

Conflict of interest: The authors have declared that no conflict of interest exists. Nonstandard abbreviations used: BCG, bacillus Calmette-Guérin; dll, delta-like; $\mathrm{mDC}$, myeloid DC; PPD, purified protein derivative.

Citation for this article: J. Clin. Invest. 119:33-46 (2009). doi:10.1172/JCI35647.
Th1 cell involvement and histologically showed an enrichment of DCs and macrophages at the granuloma sites $(5,6)$.

DCs and macrophages can respond to microbial ligands through TLRs (7), which function in the recognition of microbial components and play an important role in both the innate and adaptive immune responses (8-10). Microbial products, including Mycobacterium Ags, can activate specific TLR and induce specific gene transcription, resulting in the upregulation and secretion of select chemokines and cytokines (11-13). The involvement of TLR2, TLR4, TLR6, and TLR9 has been shown to be important in the recognition of Mycobacterium Ags in both mice and humans (14-16). TLR9 recognizes both viral and bacterial CPG-DNA motifs including those of mycobacteria, which, when bound to TLR9 on macrophages and DCs, cause their activation (17-20). In a previous study, we demonstrated that TLR9 activation is essential for the maintenance of a Mycobacterium Ag-elicited pulmonary granulomatous response; however, the exact mechanism is not known (21).

$\mathrm{CD}^{+} \mathrm{T}$ cells are essential regulators of immune responses and inflammatory diseases. Recently, IL-17-producing T cells have been described and named Th17 (22). Th17 cells are preferential producers of IL-17A and IL-17F (22) and play an essential role in host defense via protection against bacterial pathogens $(23,24)$. A combination of TGF- $\beta$ plus IL- 6 was recently shown to be essential for the initial differentiation of Th17 cells (25). Upon differentiation, IL-21 is significantly induced by the developing Th17 cells and acts in an autocrine fashion to amplify this population (26, 
27). IL-23, a p40p19 heterodimeric cytokine (28), then stabilizes the differentiated Th17 cells (29). Despite our growing knowledge of Th17 cells, the mechanism triggering IL-17 expression in these cells and their role in host defense within the context of granuloma formation remains to be determined.

Recent data have indicated that the controlled expression of Notch receptor proteins on Th cells is essential for normal T cell development and maturation (30). There are 5 mammalian ligands (delta-like 1 [dll1], dll3, dll4, Jagged-1, and Jagged-2), each of which can activate any of the 4 Notch receptors (Notch1, -2, -3, -4) (31). APCs, which express both Notch ligands and TLRs, can also induce naive $\mathrm{T}$ cell differentiation to Th1 or Th2 phenotypes with TLRligand binding (32). The connection between the TLR and Notch pathways has helped to define the complex role of APCs in the regulation of Th1/Th2 differentiation. In the presence of functional MyD88, TLR binding activates the upregulation of dll4, which causes the differentiation of naive Th cells to a Th1 phenotype. In the absence of functional MyD88, TLR pathways are inactive and constitutively expressed Jagged-1 can cause the differentiation of naive Th cells to a Th2 phenotype $(33,34)$. In contrast, precisely how Notch protein activation regulates Th17 cell phenotype and the relationship of TLRs in these processes is poorly understood.

In the present study, Th17 cytokine (IL-17A, IL-17F, IL-6, IL-21, IL-23, TGF- $\beta$ ) expression was determined in both WT and Tlr9-/mice immunized with bacillus Calmette-Guérin (BCG) and subsequently challenged with PPD-coated beads to induce lung granulomas. We demonstrate that Tlr9-deficient mice developed significantly larger granulomas with an abrogated Th17 cytokine profile and an impaired accumulation of DCs as compared with control mouse granulomas. Lung- and BM-derived DCs from $\mathrm{Tlr}^{-1-}$ mice stimulated with Mycobacterium Ags showed lower expression of both Th17 cytokines such as IL-6 and the Notch ligand dll4. We also found that BM-derived DCs but not BM-derived macrophages promoted Th17 differentiation of lung $\mathrm{CD}^{+} \mathrm{T}$ cells while both of these APCs promoted Th1 differentiation. Our results further showed that specific neutralization of dll4 during pulmonary granuloma formation induced development of larger granulomas and decreased levels of Th17-type cytokines. Moreover, in in vitro experiments, anti-dll4 Ab specifically blocked IL-17 expression, while overexpression of dll4 augmented IL-17 production. The results of this study show that dll4 influences the development of Th17 and that TLR9 deficiency leads to impaired dll4 induction, which collectively provides a key pathway for mechanistically controlling mycobacteria-dependent granulomatous responses.

\section{Results}

Tlr9 $9^{-1-}$ mice display an altered cytokine phenotype during Mycobacterium Ag-elicited pulmonary granuloma formation. To investigate the role of TLR9 on the effector stage of Mycobacterium Ag-elicited responses, mice were sensitized intratracheally with $2.5 \times 10^{5}$ CFUs of live BCG and 14 days later challenged via the tail vein with PPD-coupled beads. As shown in Figure 1, there is a striking histological (Figure 1A) and size (Figure 1B) difference between the BCG/ mycobacteria-induced lung granuloma developing in WT and that in $\operatorname{Tl} 9^{-/-}$mice. To understand the differences between pulmonary granuloma formation in $\mathrm{Tlr}^{-1-}$ and WT mice, we examined the cytokine profile in the granulomatous lungs. When compared with lungs of WT mice, granulomatous lungs of $\operatorname{Tlr}^{-/-}$mice expressed significantly lower levels of IL-17 protein (Figure 1C) as well as decreased IL-17A and IL-17F mRNA expression (Figure 1,
D and E). Moreover, the mRNA expression levels of IL-6, IL-21, and IL-23p19, which respectively support the differentiation, expansion, and stabilization of Th17 cells, were significantly reduced in $\operatorname{Tlr}^{-1-}$ mice at most time points considered (Figure $1 \mathrm{~F}$ ). Interestingly, the mRNA expression level of TGF- $\beta$ was not altered in the granulomatous lungs. We also investigated TNF- $\alpha$ and IL-10 expression, as Th17 responsiveness is amplified by TNF- $\alpha$ (35) and inhibited by IL-10 (36). As shown in Figure 1F, the mRNA expression of TNF- $\alpha$ was decreased while that of IL-10 was enhanced in Tlr $9^{-/-}$mice compared with WT mice. The protein expression data also showed significantly enhanced cytokine levels of IL-6, IL-23, and TNF- $\alpha$ in WT mice compared with Tlr9-/- mice (Supplemental Figure 1; supplemental material available online with this article; doi:10.1172/JCI35647DS1).

Impaired Th17 cell phenotype and reduced numbers of myeloid DCs in Tlr $9^{-1-}$ mice during pulmonary granuloma formation. To further characterize the changes in Th profiles that occurred between WT and Tlr $9^{-/-}$mice during granuloma formation, the populations of Th1, Th2, and Th17 cells from PPD-bead challenged mice were assessed. Flow cytometric analysis revealed that the number of IFN- $\gamma-$ and IL-4-producing $\mathrm{CD}^{+} \mathrm{T}$ cells was similar between these mice, while the number of IL-17-producing $\mathrm{CD}^{+} \mathrm{T}$ cells was severely decreased in Tlr9-deficient lungs 4 days after PPD-bead challenge (Figure 2, A and B).

Since Th cell subset differentiation and activation involves signals provided by APC populations, we hypothesized that TLR9 deletion might influence Th17 cell activity by affecting APCs. We first assessed numbers of APCs in granulomatous lungs by examining $\mathrm{CD}_{4} 5^{+}$leukocytes 4 days after PPD-bead challenge. Compared with WT mice, the number of myeloid DCs (mDCs) $\left(\mathrm{CD} 11 \mathrm{~b}^{+} \mathrm{CD} 11 \mathrm{c}^{+}\right)$was significantly decreased in $\operatorname{Tl} 9^{-/-}$mice, while plasmacytoid DC $\left(\mathrm{B} 220^{+} \mathrm{CD} 11 \mathrm{c}^{+}\right)$numbers were similar in both sets of mice (Figure $2 \mathrm{C}$ ). In contrast, the number of $\mathrm{F} 4 / 80^{+}$macrophages was significantly increased in $\operatorname{Tlr}^{-1-}$ mice (Figure $2 \mathrm{C}$ ). However, the number of Gr-1 ${ }^{\text {hi }}$ neutrophils was similar in both groups of mice (Figure 2C). These results were also supported by confocal immunofluorescent analysis, which showed fewer $\mathrm{CD} 11 \mathrm{c}^{+}$staining cells (red) and an increase in F4/80 ${ }^{+}$cells (green) associated with the local lung granulomas in $T / r 9^{-/-}$mice (Figure 2D).

To further determine the reason why the number of DCs was reduced in $\mathrm{Tlr}^{-/-}$mice, we investigated chemokine expression during granuloma formation. As shown in Figure 2E, the mRNA expression levels of CCL20 and CCL22 were significantly decreased in Tlr $9^{---}$mice. Interestingly, CCL20 and CCL22 have been shown to play a crucial role in the recruitment of immature DCs into inflammatory lesions $(37,38)$.

Tlr9-1- mice display altered mDC mobilization and dll4 Notch ligand expression during Mycobacterium Ag-elicited pulmonary granuloma formation. We next examined possible functional changes in DCs from $\mathrm{Tl}_{\mathrm{O}} \mathrm{O}^{-1-}$ mice during pulmonary granuloma formation. We initially assessed the cytokine profile of DCs recovered from granulomatous lungs. In these experiments, lung CD $11 \mathrm{~b}^{+} \mathrm{CD} 11 \mathrm{c}^{+} \mathrm{DCs}$ were sorted on a FACSAria cell sorter (BD Biosciences). After DC purification, more than $95 \%$ of the cells were CD $11 b^{+} C D 11 c^{+}$. DCs from Tlr9-deficient lungs harvested at day 4 after PPD-bead challenge showed lower mRNA levels for IL-6, IL-12p40, and IL-23p19 compared with those from WT mice (Figure 3A). In contrast, the Tlr9-deficient DCs showed enhanced IL-10 mRNA levels (Figure 3A). We also measured transcripts for Notch ligands dll4 and Jagged-1, which can differentially affect Th differentiation/activation. 
A

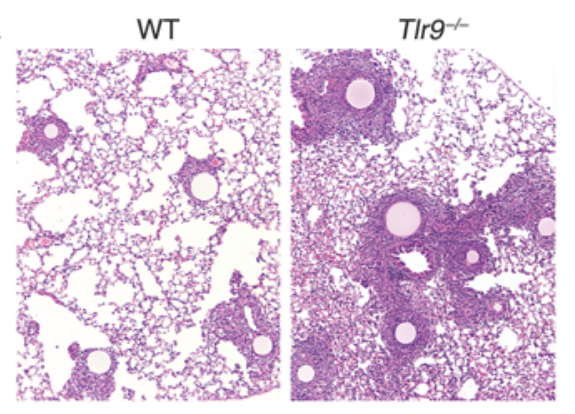

B

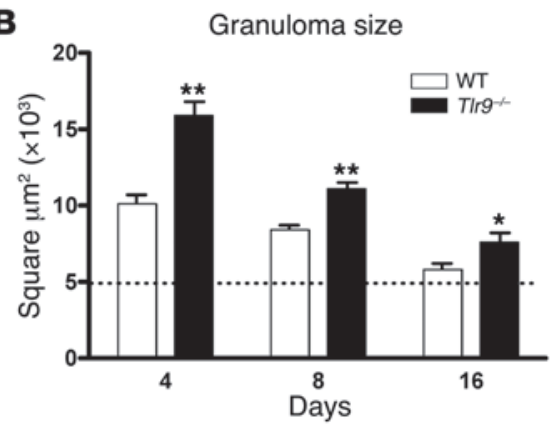

C

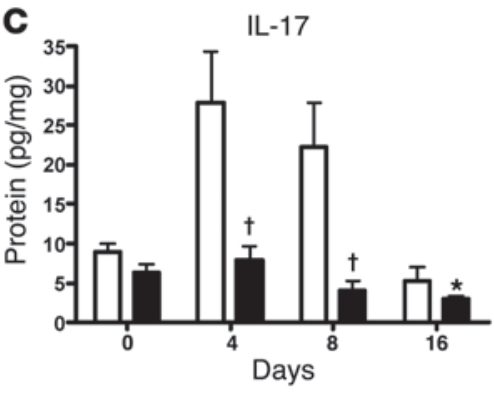

$\mathbf{F}$
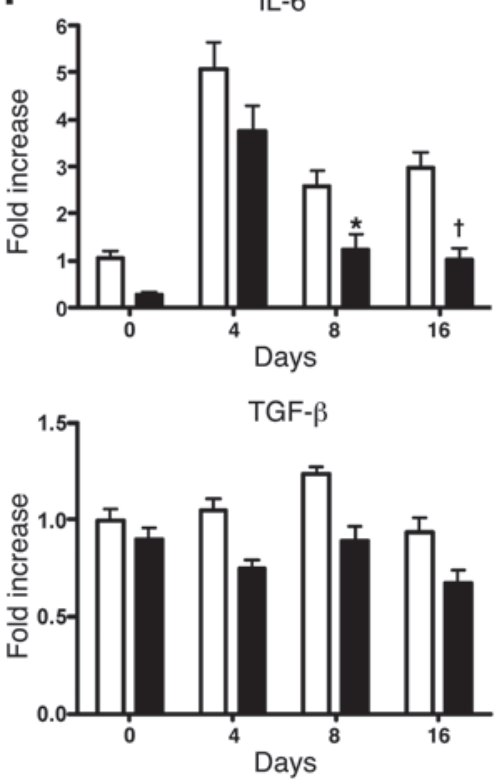

D
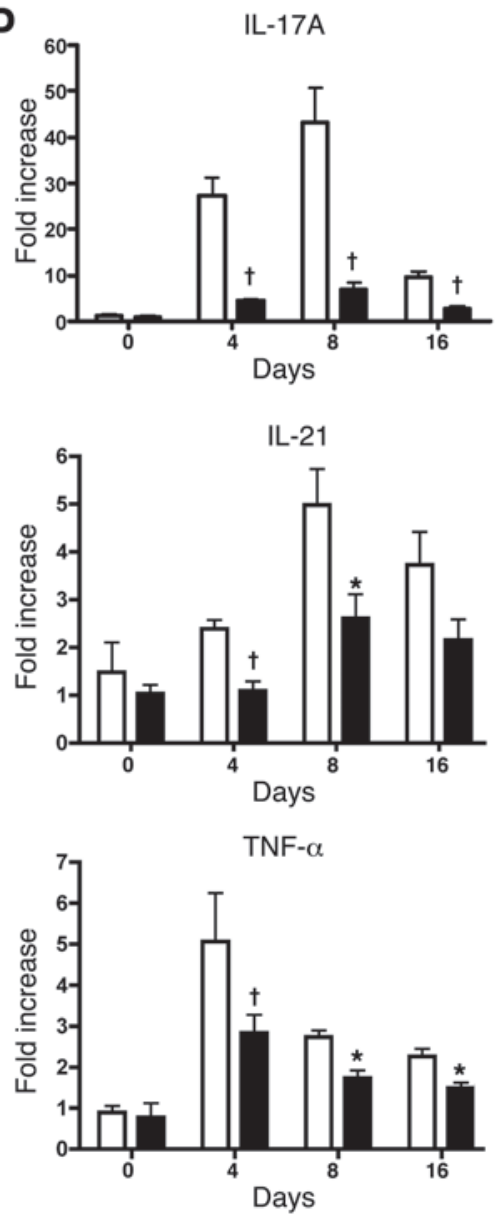

E

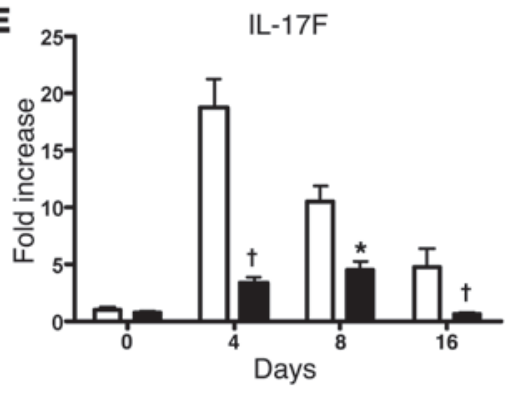

IL-23p19
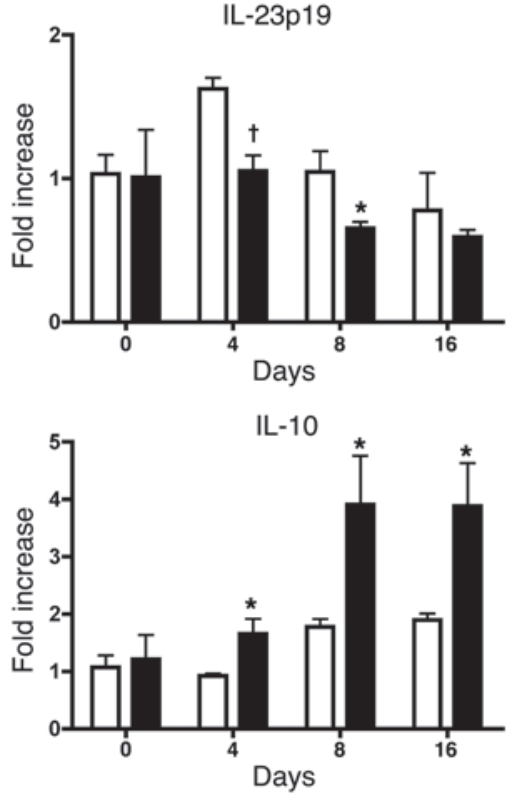

Figure 1

TIr9-/- mice showed larger pulmonary granulomas and impaired Th17 cytokine levels. All panels compare parameters between WT and TIr9-/mice. (A) Lung tissues were histologically analyzed by H\&E at day 4 after initiation of lung granuloma. Original magnification, $\times 100$. (B) Kinetic analysis of the development of lung granulomas using morphometric analysis of the evolving lung lesions in WT and TIr9-/- mice. Dotted line represents mean granuloma cross-sectional area $\left(\mu \mathrm{m}^{2}\right) \pm$ SEM. ${ }^{*} P<0.05 ;{ }^{* \star} P<0.001$. (C) Protein levels of IL-17 measured in whole lungs using a Luminex system. Data shown are mean \pm SEM and are from a representative experiment of 4 independent experiments. ${ }^{\dagger} P<0.01$. (D-F) Quantitative real-time PCR (TaqMan) was performed to measure the transcript levels of IL-17A (D), IL-17F (E), IL-6, IL-21, IL-23p19, TGF- $\beta$, TNF- $\alpha$, and IL-10 (F) in whole lungs. Data shown are mean \pm SEM and are from a representative experiment of 4 independent experiments. Each time point indicates at least 4-6 mice per group.

In particular, dll4 has been reported as an important factor for differentiation of Th cells (33). As shown in Figure 3B, levels of dll4 mRNA were significantly lower in $\operatorname{Tr} 9^{-/-}$mice, whereas Jagged-1 mRNA levels were similar between WT and Tlr9-/- lung DCs (Figure 3B). Notch ligands dll1, dll3, and Jagged-2 were below detection levels of our assay (data not shown). In agreement with the DC transcript data, we also found that dll4 mRNA levels were increased in whole granulomatous lungs from WT mice over the study period (Figure 3C), while the expression of dll1, Jagged-1, and Jagged-2 was unchanged. dll3 was not detected here or in any experiments described below. In contrast, dll4 mRNA levels were profoundly reduced in whole granulomatous lungs from 

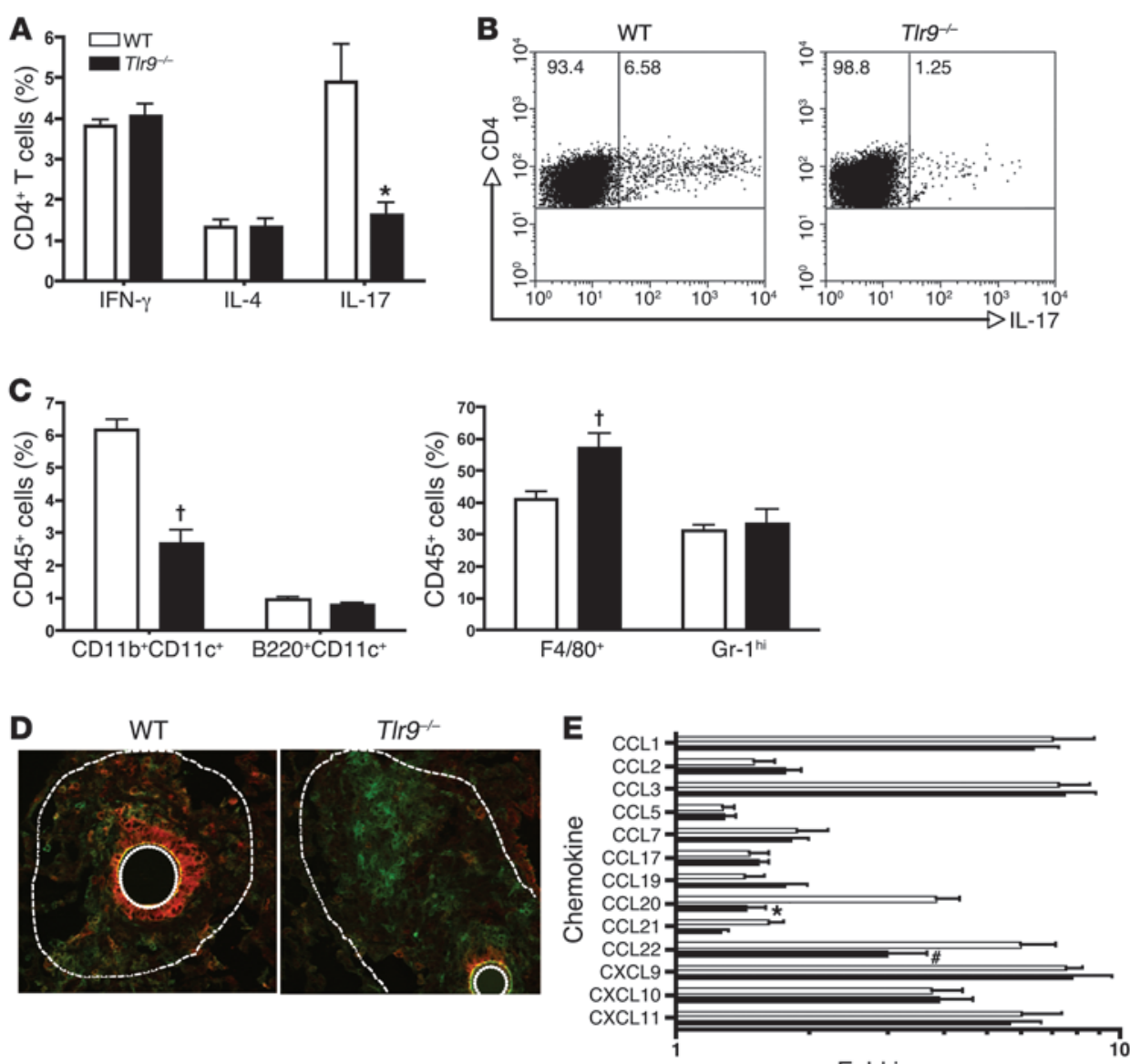

Figure 2

Impaired Th17 cell phenotype and migration of mDCs in TIr9-/- mice during pulmonary granuloma formation. (A) FACS analysis of intracellular staining of $\mathrm{CD}^{+}{ }^{+}$cells for IFN- $\gamma$, IL-4, and IL-17. Data represent mean \pm SEM. ${ }^{*} P<0.01$ compared with WT mice. Experiments were performed 3 times with similar results. (B) FACS profile of CD4+ $\mathrm{T}$ cells from lungs was analyzed by intracellular staining of IL-17. Displayed in the dot plots are cells gated on CD4+ lymphocyte population by forward scattering and side scattering. The numbers in the dot plots are percentages of IL-17- cells (left gate) and IL-17+ cells (right gate) in the CD4+ lymphocyte population. Shown are representative FACS plots from a total of 4 mice per group. (C) FACS analysis of lung DCs, macrophages, and neutrophils isolated from day 4 PPD-bead challenged mice $(n=4)$. ${ }^{\dagger} P<0.02$ compared with WT mice. Data shown are mean \pm SEM and are from a representative experiment of 2 independent experiments. (D) Confocal immunofluorescent examination of pulmonary granulomas showed fewer CD11 $\mathrm{c}^{+}$cells (red) and increased F4/80+ cells (green) in TIr9-/- mice. Shown are representative sections from 1 mouse of 4 per group. Dotted lines indicate the areas containing a PPD bead and the granuloma. Original magnification, $\times 200$. (E) Quantitative real-time PCR (TaqMan) was performed to measure the transcript levels of chemokine in whole lung at day 4 after the initiation of lung granuloma. $\# P<0.05$; $P<0.01$. Data shown indicate mean \pm SEM and are from a representative experiment of 3 independent experiments. Each time point indicates at least $4-6$ mice per group.

Tlr9 ${ }^{-/-}$mice compared with WT mice over the study period (Figure $3 \mathrm{D})$. As with the purified DCs, there were no significant differences in Notch ligand dll1, Jagged-1, and Jagged-2 mRNA levels in whole granulomatous lungs from WT and $T l r 9^{-/-}$mice (data not shown). In addition, flow analysis demonstrated that lung mDCs $\left(\mathrm{CD} 11 \mathrm{~b}^{+} \mathrm{CD} 11 \mathrm{c}^{+}\right)$expressed lower protein levels of dll4 in Tlr $9^{-1-}$ mice compared with WT mice (Figure $3 \mathrm{E}$ ).

BM-derived DCs from Tlr9-/- mice produce lower levels of Th17-related cytokines with impaired dll4 expression after BCG stimulation. The above studies suggested that APCs from $\operatorname{Tlr}^{-1-}$ mice were func-
Fold increase

tionally altered at least with regard to specific cytokine and dll4 Notch ligand expression. To determine whether the TLR9 signaling pathway regulates dll4 Notch ligand expression, we stimulated BMderived DCs with CPG for 6 hours, harvested cells, and assessed samples for mRNA expression. The mRNA level of dll4 was dramatically and significantly increased compared with that of other Notch ligands, dll1, Jagged-1, and Jagged-2, in BM-derived DCs from WT mice (Figure 4A).

To further investigate whether dll4 might influence T cell activation, we generated APCs from WT and $\operatorname{Tl} 9^{-/-} \mathrm{BM}$ for use in a cytokine elicitation assay. Since macrophages and DCs are significant cellular components of granulomas as well as potential APCs, we generated both DCs and macrophages from BM of WT and Tlr9-/- mice. These were first characterized by their ability to express specific cytokine and Notch ligand mRNA. DCs and macrophage cultures were infected with BCG $(\mathrm{MOI}=0.1$ or 1$)$ for 6 hours. Cells were harvested and samples assessed for mRNA expression. The BCG-elicited transcripts for IL-6, IL-12p40, IL-23p19, and TNF- $\alpha$ were significantly lower in BM-derived DCs from $\mathrm{Tlr}^{-/-}$mice when compared with BM-derived DCs from WT mice (Figure 4B), while IL-10 transcripts were undetectable (data not shown). In contrast, BM-derived macrophages from $\mathrm{Tlr}^{-/-}$mice and WT mice showed no significant differences in expression levels of IL-12p40, IL-6, and IL-23p19 mRNA (Figure $4 C)$. However, TNF- $\alpha$ transcripts were significantly lower, while IL-10 mRNA levels were enhanced in BM-derived macrophages from Tlr9 ${ }^{-/-}$mice (Figure 4C).

These macrophages did not show alternatively activated macrophage characteristics such as increased FIZZ-1 and Arginase-1 expression, and there was no significant difference in the expression of these in BM-derived macrophages from WT and Tlr9-/mice during BCG stimulation (Supplemental Figure 2). In regard to Notch ligands, dll4 transcript induction was significantly decreased in $\operatorname{Tl} 9^{-/-}$DCs with no effect on Jagged-1 transcripts (Figure 4B). In contrast, no significant difference in dll4 expression was observed in BCG-challenged or -unchallenged macrophages derived from WT or Tlr9-/- mice (Figure 4C). Also, no 
A
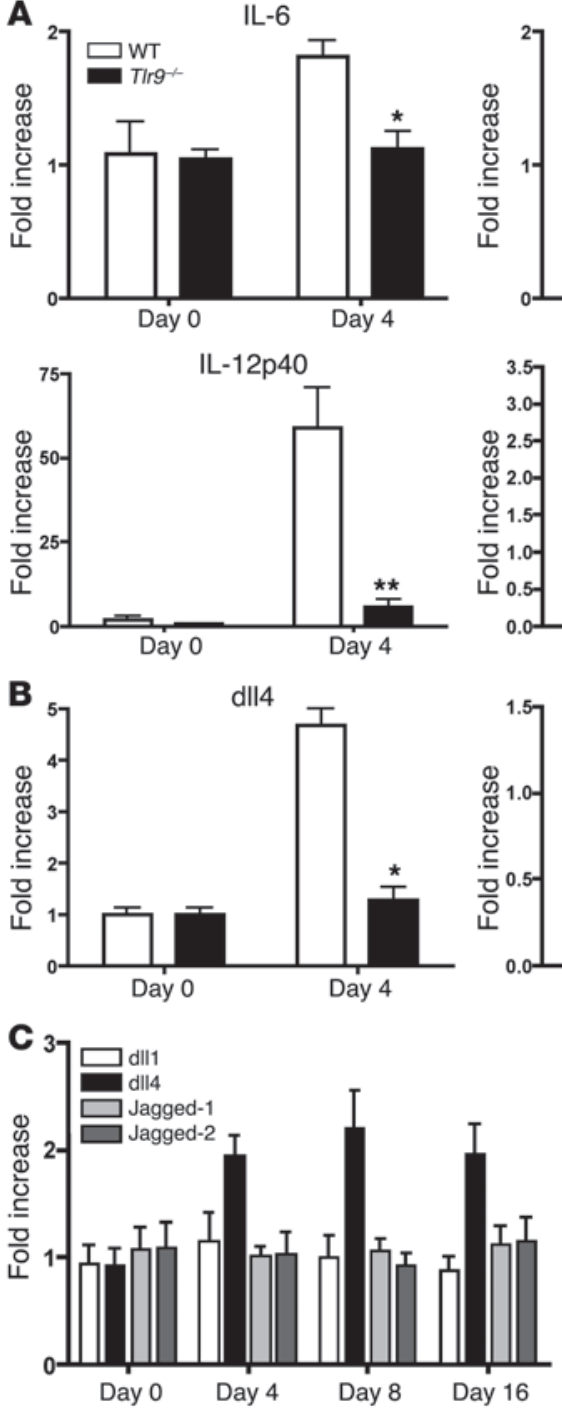

IL-10
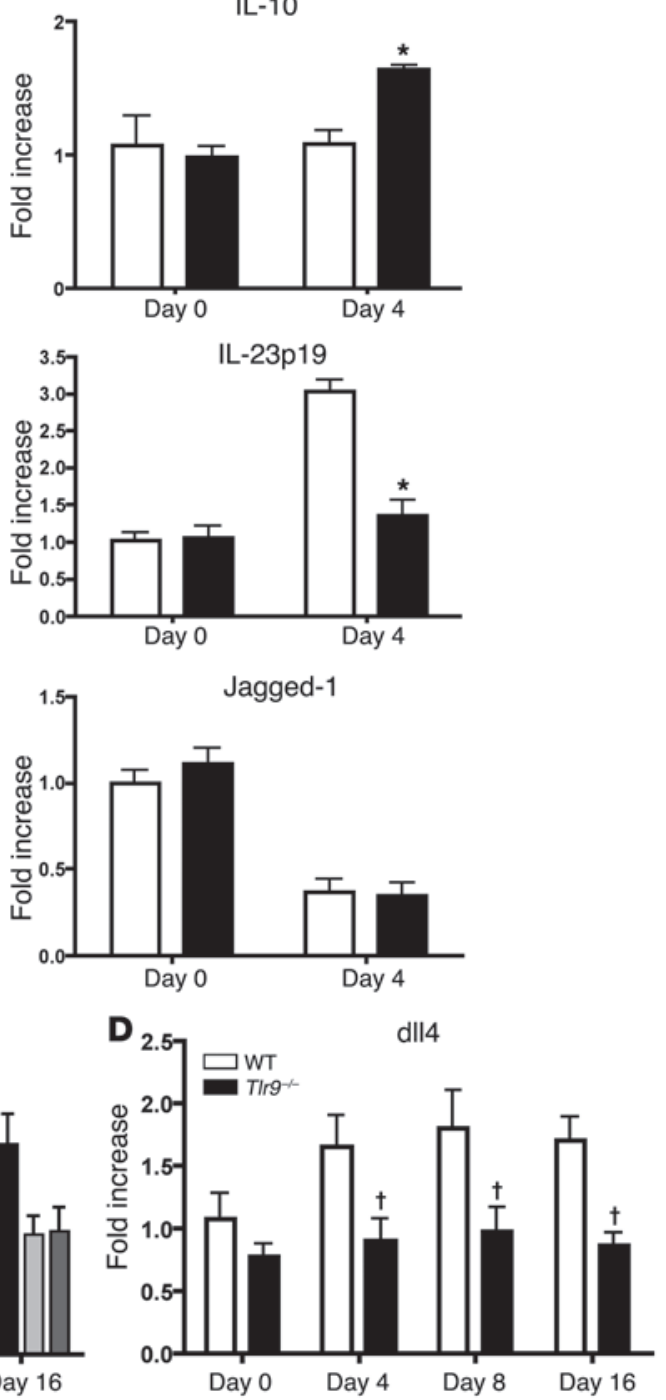

E
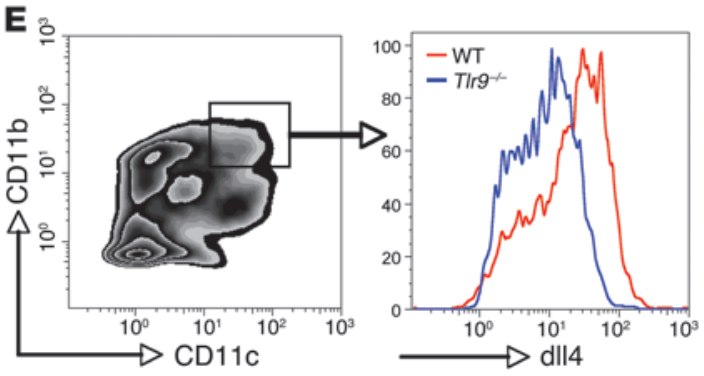

Figure 3

Lung DCs from TIr9-/- mice exhibit decreased expression levels of both Th17-related cytokine and dll4. Lung mDCs $\left(C D 11 b+C D 11 c^{+}\right)$were purified from granulomatous lungs using cell sorter at days 0 and 4 after PPD-bead challenge. mRNA levels were quantitated as described in Methods. (A) Gene expression levels of IL-6, IL-10, IL-12p40, and IL-23p19 in lung DCs. ${ }^{*} P<0.03$; ${ }^{* *} P<0.01$ compared with lung DCs from WT mice. (B) Gene expression levels of Notch ligands in lung DCs. ${ }^{*} P<0.03$ compared with lung DCs from WT mice. (C) Gene expression levels of Notch ligands (dll1, dll4, Jagged-1, and Jagged-2) in whole lungs from WT mice during granuloma formation. (D) Gene expression of dll4 in whole lungs from WT and Tlr9-/- mice during granuloma formation. ${ }^{\dagger} P<0.05$. (E) The level of dll4 in lung $\mathrm{mDCs}\left(\mathrm{CD} 11 \mathrm{~b}^{+} \mathrm{CD} 11 \mathrm{c}^{+}\right)$from WT or $\mathrm{Tlr9}^{-/-}$mice was determined with flow cytometry using a specific polyclonal $\mathrm{Ab}$ against dll4. Significant differences in MFI were seen when dll4 protein expression in DCs from WT mice was compared with $\mathrm{Tlr}^{-/-}$at day 4 . Data shown indicate mean \pm SEM and are from a representative experiment of 3 independent experiments. Each time point represents at least 4 mice per group. transcript expression of the Notch ligands dll1, dll3, and Jagged-2 was detected in BM-derived macrophages (data not shown). Flow analysis data showed BM-derived DCs express lower protein levels of dll4 in $T l{ }^{-1-}$ mice compared with in WT mice during BCG stimulation (Figure 4D).

A bioplex proteomic assay or ELISA system was used to compare the cytokine protein phenotypes for IL-12p40, IL-6, TNF- $\alpha$, IL-10, and IL-23 in BCG-stimulated BM-derived DCs and macrophages recovered from $\mathrm{Tl}_{\mathrm{r}} \mathrm{9}^{-/-}$and WT mice. Our results showed a decrease in IL-12p40, IL-6, and IL-23 protein production in
BM-derived DCs from $\operatorname{Tl} 9^{-/-}$mice compared with those from WT mice, while BM-derived macrophages from both WT and Tlr $9^{-/-}$mice produced comparable low levels of IL-12p40 and similar amounts of IL-6 and IL-23 (Supplemental Figure 3, A, B, and $\mathrm{E}$ ). We also investigated TNF- $\alpha$ and IL-10 protein production from BM-derived macrophages, which were the main source of these cytokines; interestingly, macrophages from the $\mathrm{Tlr}^{-/-}$mice expressed significantly lower levels of TNF- $\alpha$ and significantly higher levels of IL-10 compared with macrophages from WT mice (Supplemental Figure 3, C and D). 

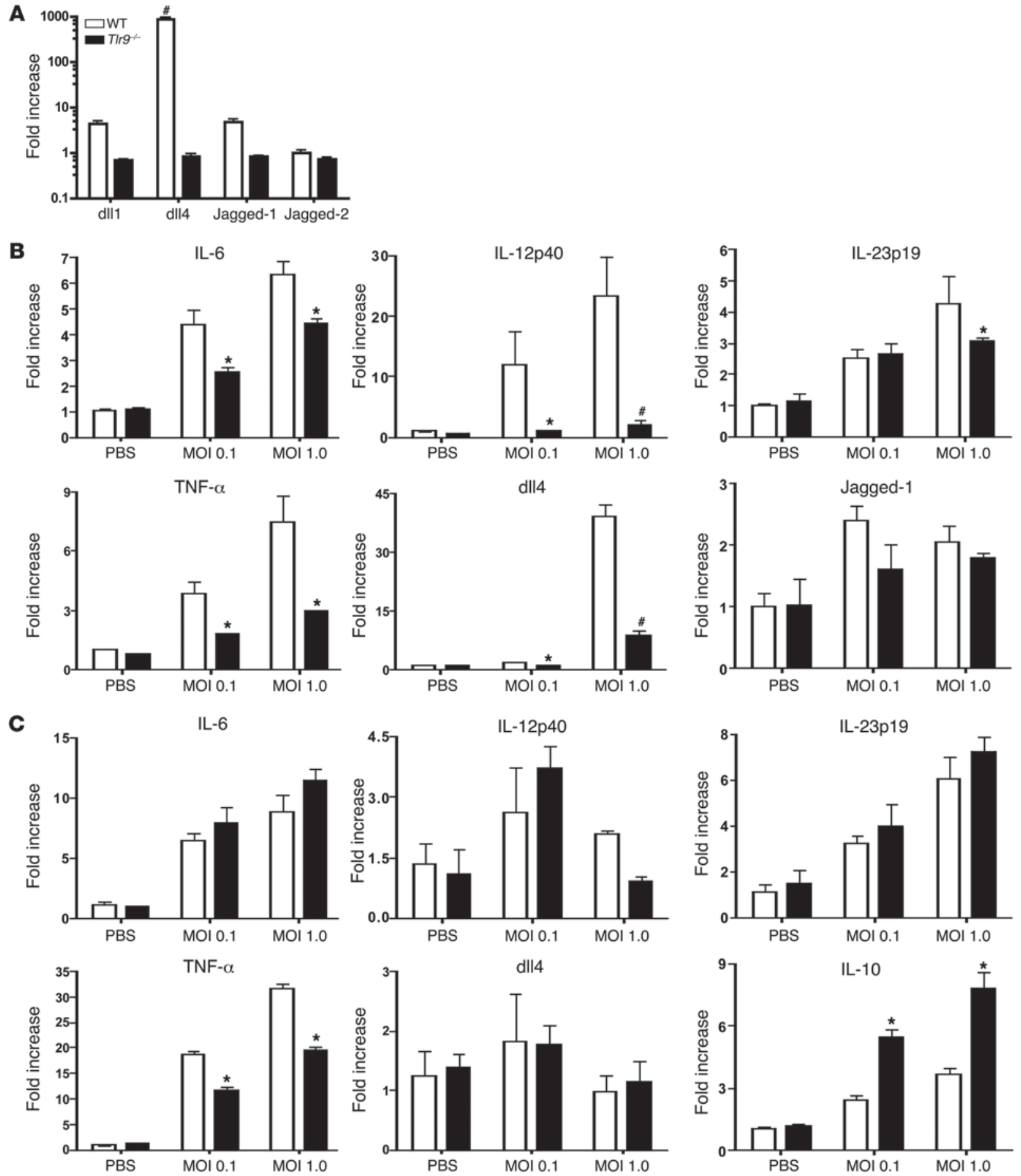

D
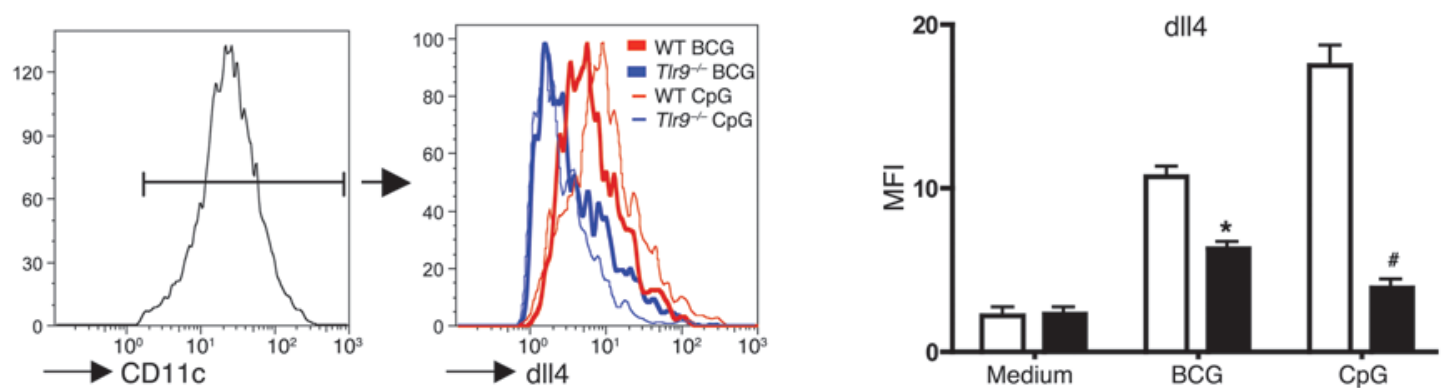


\section{Figure 4}

BM-derived DCs but not BM-derived macrophages from T/r9-/- mice exhibit decreased expression levels of Th17-related cytokines and dll4. (A) BM-derived DCs from WT and T/r9-/- mice were stimulated with CpG $(1 \mu \mathrm{M})$ for 6 hours, and then quantitative real-time PCR was performed and expression levels of Notch ligands were evaluated. ${ }^{*} P<0.01$. (B) BM-derived DCs and (C) BM-derived macrophages from TIr9--- and WT mice were stimulated with $B C G(\mathrm{MOI}=0,0.1$, or 1.0$)$ for 6 hours, then quantitative real-time PCR was performed to assess mRNA expression for indicated cytokines. ${ }^{*} P<0.05$. (D) BM-derived DCs from WT or $\mathrm{TIr} \mathrm{I}^{-/-}$mice were stimulated with $\mathrm{BCG}(\mathrm{MOI}=1.0)$ or $\mathrm{CpG}(1 \mu \mathrm{M})$ for 24 hours. Flow cytometry was performed using a specific polyclonal $\mathrm{Ab}$ against dll4. Bars within histogram spans indicate cells gated on $\mathrm{CD} 11 \mathrm{C}^{+}$populations. Significant differences in MFI were seen when dll4 protein expression in DCs from WT mice was compared with that in DCs from TIr9-/- mice at day 4. Data shown indicate mean \pm SEM and are from a representative experiment of 3 independent experiments. Each time point represents at least 4 mice per group.

Specific neutralization of IL-6 during Mycobacterium Ag-elicited pulmonary granuloma formation increases granuloma size with impaired Th17 cells. IL-6 plays an important role in the differentiation of Th17, and $\operatorname{Tr} 9^{-1-}$ mice showed lower expression of IL-6 in both whole lungs and DCs during granuloma formation when compared with WT mice. To further examine the distinct role of IL-6, we blocked its activity with anti-IL-6 Abs. This treatment in WT mice recapitulated the $\operatorname{Tlr}^{-1-}$ state, resulting in significantly larger granulomas (Figure 5, A and B), and abrogated IL-17 production (Figure $5 \mathrm{C})$. Specifically, the treatment of mice with anti-IL-6 Ab led to a significant decrease not only in IL-17A and IL-17F expression but also in IL-21 expression (Figure 5D). Moreover, flow cytometric analysis revealed that the number of IFN- $\gamma$ - and IL-4-producing $\mathrm{CD}^{+} \mathrm{T}$ cells was similar between anti-IL-6-treated mice and control mice, while the number of IL-17-producing $\mathrm{CD}^{+} \mathrm{T}$ cells was significantly decreased in anti-IL-6 Ab-treated lungs 4 days after PPD-bead challenge (Figure 5, E and F).

BM-derived DCs but not macrophages promote the expression of IL-17 from lung T cells during a BCG-induced immune response. We next compared BCG-stimulated DCs and macrophages with respect to their influence on lung $\mathrm{CD}^{+}{ }^{+} \mathrm{T}$ effector cell expression using a coculture cytokine elicitation assay. As shown in Figure 6, both BM-derived DCs and BM-derived macrophages were able to induce IFN- $\gamma$ production by $\mathrm{CD}^{+} \mathrm{T}$ cells isolated from the granulomatous lungs of either WT or Tlr9-/- mice (Figure 6, A and C). However, IFN- $\gamma$ production was significantly less when the DCs or macrophages were derived from $\mathrm{Tlr}^{-1-}$ mice (Figure 6, A and C). Interestingly, only BM-derived DCs were capable of inducing IL-17; however, DCs recovered from $\operatorname{Tl} 9^{-1-}$ mice were impaired in this capacity, as compared with DCs from WT mice. (Figure 6, B and D).

dll4 regulates mycobacteria-elicited Th17 effector cell expression. Our studies of BM-derived APCs suggested that CD $11 \mathrm{c}^{+}$mDCs but not macrophages supported the Th17 effector response. Moreover, $\operatorname{Tl} 9^{-/-}$mDCs from either granulomatous lungs or from BM showed a consistent defect in dll4 Notch ligand expression, pointing to a possible relationship among TLR9, dll4, and Th17 effector expression. To test this in vivo, we blocked dll4 functionality in WT mice by passive immunization with Ab directed against murine dll4. This treatment recapitulated the $\operatorname{Tl} 9^{-/-}$state, resulting in significantly larger granulomas (Figure 7, A and B), reduced $\mathrm{mDC}$ elicitation (Figure $7 \mathrm{C}$ ), altered IL-17 protein levels (Figure 7D), and lower Th17-related cytokine transcript expres- sion (Figure 7E). Specifically, the treatment of mice with anti-dll4 led to a significant decrease in both IL-17A and IL-17F expression compared with that in control mice (Figure 7E) and decreased the expression of Th17-related cytokines IL-6 and IL-21 (Figure $7 \mathrm{E})$. There was no significant difference in IL-23p19, TNF- $\alpha$, and TGF- $\beta$ expression between treated and control groups (data not shown). To further characterize the effect of dll4 blockade during granuloma formation, we evaluated DC numbers in the lungs of control and anti-dll4-treated mice by flow cytometry 4 days after PPD bead challenge. As shown in Figure 7C, the number of mDCs $\left(\mathrm{CD} 11 \mathrm{~b}^{+} \mathrm{CD} 11 \mathrm{c}^{+}\right)$was significantly decreased in anti-dll4-treated mice. To determine whether the decreased numbers of DCs seen in granulomas from anti-dll4-treated mice were due to impaired recruitment of DCs, we examined the chemokine expression in the lung. As shown in Figure 7F, the treatment of mice with anti-dll4 led to decreased expression in the lungs of DC and Th17 recruitment chemokine CCL20.

dll4 specifically regulates activation of Th17 cytokines during immune response. To directly test the effect of dll4 on the Th phenotype, we performed an in vitro lung $\mathrm{CD}^{+} \mathrm{T}$ cell cytokine expression assay with BCG-stimulated BM-derived DCs with either depletion or addition of dll4. As shown in Figure 8, A and B, addition of recombinant dll4 augmented IL-17 production from $T$ cells isolated from BCG-challenged lungs concomitant with BCG-treated BM-derived DCs while having no effect on IFN- $\gamma$ production. Additionally, anti-dll4 Ab added to similar cultures significantly decreased IL-17 production with no effect on IFN- $\gamma$ production (Figure 8, C and D). To determine whether the effect of dll4 on IL-17 and IFN- $\gamma$ was Ag specific, we evaluated the production of these cytokines by splenic CD4 ${ }^{+} \mathrm{T}$ cells harvested from DO11.10 mice, which have OVA-specific TCR, that were cocultured with BM-derived DCs from WT or Tlr9-/- mice (Figure 8, E and F). No significant differences in the production of IFN- $\gamma$ and IL-17 were seen in $\mathrm{T}$ cells cocultured with BM-derived DCs from WT or $\operatorname{Tlr}^{-1-}$ mice after stimulation with only OVA, underscoring the importance of DC-derived TLR9 activation, as these APCs engage $T$ cell activation events.

Schematic of TLR9/Notch ligand (dll4)/Th17 phenotype pathway in the evolution of mycobacteria-dependent lung granuloma. As summarized in the schematic found in Figure 9, DCs via a TLR9/dll4 network are instrumental in the differentiation of Th17 cells during mycobacteria-dependent granuloma formation. Granulomas in $\operatorname{Tlr}^{-1-}$ mice present with decreased numbers of both Th17 cells and mDCs, yet they exhibit an increase in the elicitation of granuloma macrophages and an overall exacerbation of the size of the developing lung lesion. In addition, the granulomatous lungs of the Tlr9-1- mice possess impaired levels of dll4 and the chemokines CCL20 and CCL22 as well as lower levels of IL-17 and the family of Th17 related cytokines, as compared with WT mice. Collectively, the data support a key contribution of DC-dependent TLR9 and dll4 expression to the development of a Th17 phenotype during mycobacteria-directed immune responses.

\section{Discussion}

The present investigation represents what we believe is the first analysis of cell-mediated Th17-related pulmonary Mycobacterium Ag-elicited granuloma formation in mice with targeted disruption of the TLR9 gene and defines a role for TLR9 in the induction of dll4 and Th17 expression using both in vivo and in vitro approaches. Our results demonstrate that dll4 supports Th17 
A

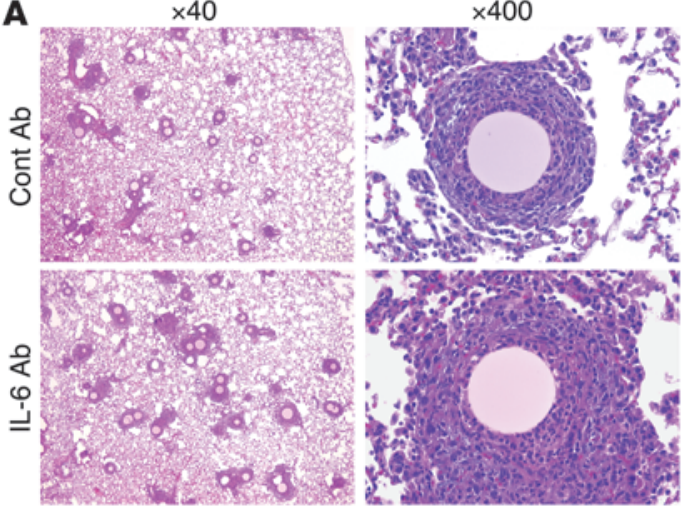

B
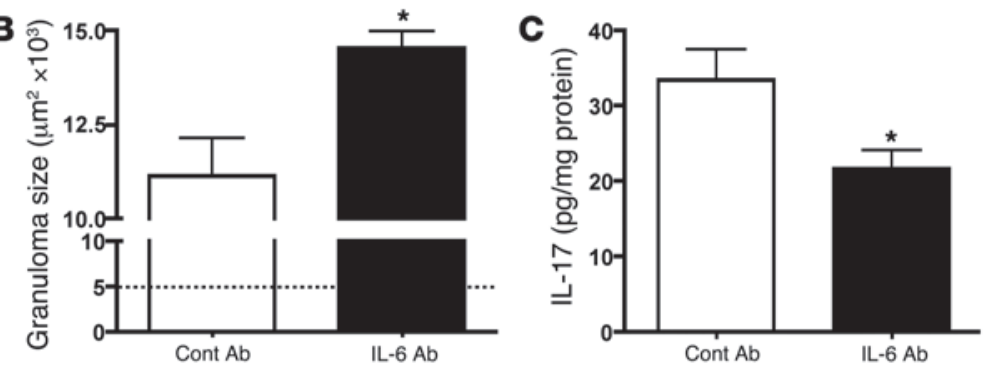

D

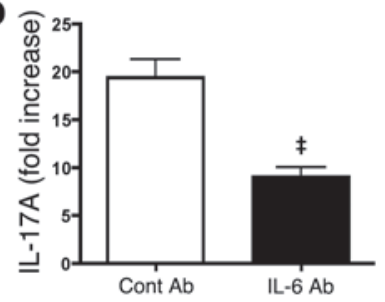

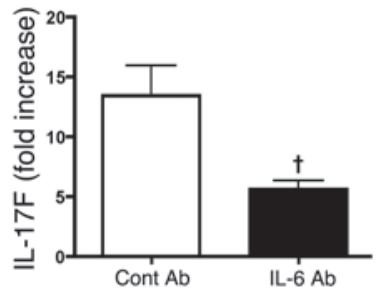

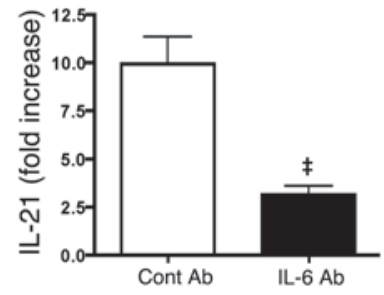

$\mathbf{F}$

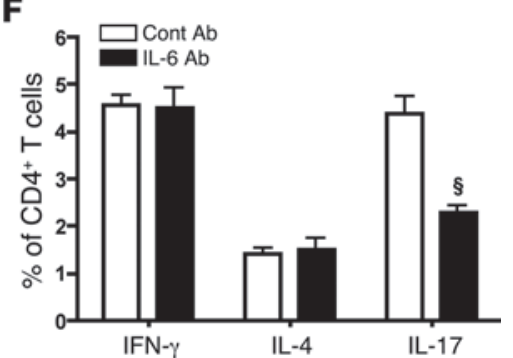

\section{Figure 5}

Blockage of IL-6 increases granuloma size and abrogates Th17 phenotype. (A) Histological appearance of pulmonary granulomas from WT mice treated with either control Abs (Cont Ab) or anti-IL-6 Abs at day 4 after granuloma development. H\&E staining. Original magnification, $\times 40 ; \times 400$. (B) Morphometric analysis of pulmonary granuloma size from mice treated with either control Abs or anti-IL-6 Abs 4 days after initiation of lung granuloma development. ${ }^{*} P<0.01$ compared with control. (C) Protein levels of IL-17 measured in whole lungs using a Luminex system. (D) Quantitative real-time PCR (TaqMan) was performed to measure the transcript levels of IL-17A, IL-17F, and IL-21 in whole lungs. ${ }^{\dagger} P<0.03$; $¥ P<0.01$ compared with control. (E) FACS profile of CD4 ${ }^{+} \mathrm{T}$ cells from lungs was analyzed by intracellular staining of IL-17. Displayed in the dot plots are cells gated on CD4+ lymphocyte population by forward and side scattering. Numbers are percentages of IL-17- cells (left gate) and IL-17+ cells (right gate) in the CD4+ lymphocyte population. Shown are representative FACS plots from a total of 4 mice per group. (F) FACS analysis of intracellular staining of $\mathrm{CD} 4^{+}$cells for IFN- $\gamma$, IL-4, and IL-17. $\$ P<0.02$ compared with control. Data represent mean \pm SEM. Experiments were performed 3 times with similar results.

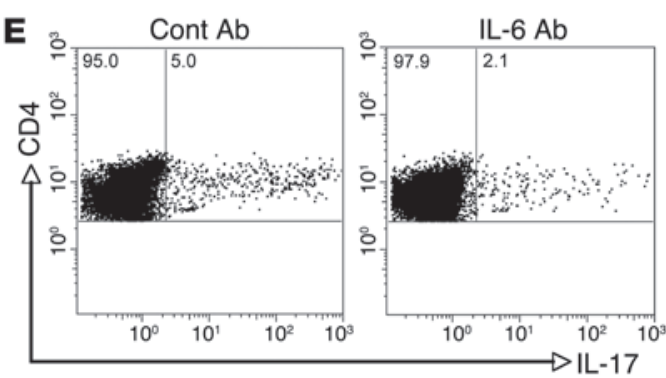

effector function and the TLR9 signaling pathway plays a central role by upregulating dll4 in response to Mycobacterium Ags. Our studies demonstrate that Tlr9-deficient mice exhibited significantly larger granuloma formation with an accompanied impaired Th17-like response. The observed histologic alterations in lung granuloma development in $\operatorname{Tr} 9^{-1-}$ mice coincided with a significant decrease in lung mDCs, which are crucial to the differentiation of Th17 cells, as well as decreased levels of dll4 on DCs when compared with those of WT mice.

We have utilized a well-established model of granulomatous lung inflammation that employs the embolization of sepharose beads coated with PPD from $M$. tuberculosis into previously immunized BCG mice. This model possesses many of the histologic characteristics observed in Mycobacterium infections (21). It is likely that TLRs are mechanistically involved in this process, as DNA from
BCG has been shown to have immunostimulatory properties (39) due to the binding of unmethylated bacterial DNA CPG motifs to host cell TLR9 $(17,40)$, which causes the activation of innate immune cells $(19,20)$. Our studies showed reduced expression of IL-17 in Tlr9-/- mice as well as larger granuloma formation when compared with WT mice, suggesting the pivotal role of TLR9 in driving the Th17-mediated immune response to Mycobacterium Ags. The involvement of IL-17 in immune response against intracellular bacteria Mycobacterium bovis BCG or M. tuberculosis has been suggested from the analysis of IL-23-deficient mice (41). IL-23 shares a p40 subunit with IL-12, a Th1-inducing cytokine (42). However, IL-12 and IL-23 are, respectively, DC- and macrophage-derived heterodimeric cytokines with distinct functions and structures $(43,44)$. IL-12 preferentially induces $T$ cells to produce IFN- $\gamma$, thereby activating phagocytes to kill intracellular patho- 

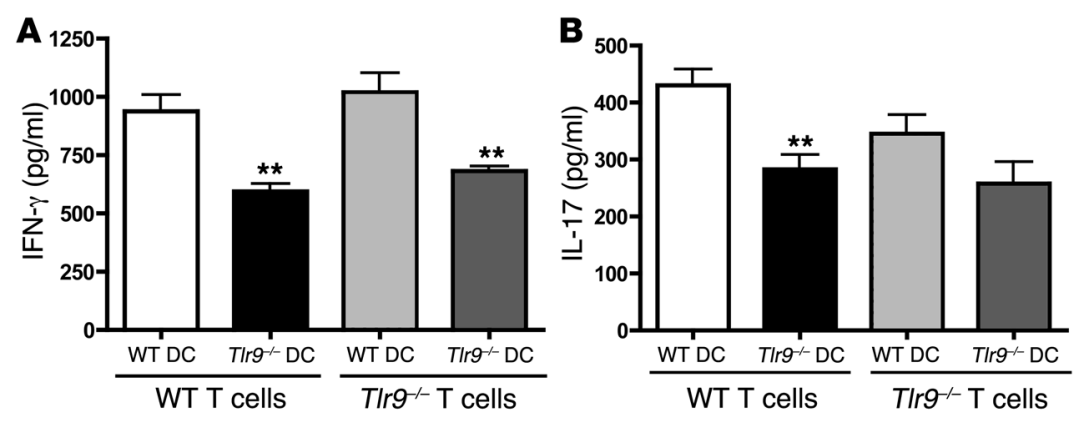

\section{Figure 6}

BM-derived DCs but not BM-derived macrophages promote the differentiation of Th17 cells from $T$ cells restimulated with $B C G$. T cells were isolated from lungs of either WT or TIr9-/- mice and restimulated with DCs (A and $\mathbf{B})$ or macrophages ( $\mathbf{C}$ and D) isolated from WT or T/r9-/- mice, as indicated. (A) IFN- $\gamma$ production. ${ }^{* *} P<0.01$. (B) IL-17 production. (C) IFN- $\gamma$ production. ${ }^{*} P<0.05$. (D) IL-17 production. Data shown indicate mean \pm SEM and are from a representative experiment of 3 independent experiments. Each point had 4 mice per group.
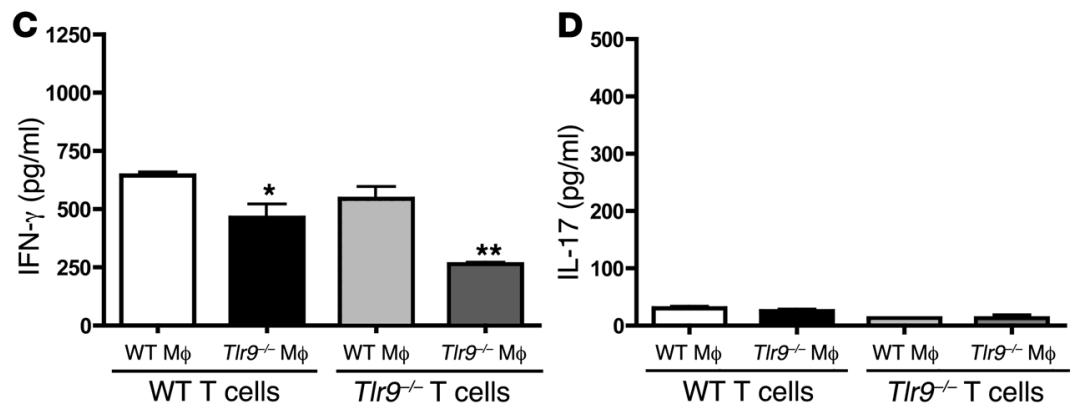

gens. In contrast, IL-23 promotes chronic inflammatory responses such as those observed in EAE (45) and collagen-induced arthritis (46), largely by stimulating T cells to produce IL-17 $(47,48)$.

Although IL-23 appeared to be required for Th17-mediated immunopathology, more recent reports indicate that it is not required for Th17 commitment and that the concomitant activity of IL- 6 and TGF- $\beta$ appears to be necessary for initiation of Th17 differentiation (25). However, IL-23 does appear to be important for amplifying and/or stabilizing the Th17 phenotype (49). The IL-12/23p40-deficient mice, which lack both IL-12 and IL-23, were more susceptible to microbial infection than IL-12p35-deficient mice, which lack only IL-12 (41). In contrast, IL-23p19-deficient mice, which lack only IL-23, showed normal protective immunity against Mycobacterium Ags (50). These studies are consistent with our data showing that enhanced granuloma formation with decreased IL-17 production in lungs may be correlated with a reduction in both IL-12p40 and IL-23p19 expression on DCs in Tlr9-deficient mice. Recent studies have shown that IL-17 does not play a significant protective role during a primary immune response; however, the presence of $\mathrm{T}$ cells capable of producing IL-17 during a secondary immune response does contribute to protection against Mycobacterium Ag challenge (51-53). These investigations suggest that Mycobacterium Ag-specific Th17 cells are potentially a new class of effector $T$ cells that respond to mycobacterial challenge. The published reports cited above agree with our findings, which show that IL-17 expression and the Th17 population in lungs receiving only a primary BCG infection are identical between WT and Tlr9-/- mice (data not shown). Interestingly, both the expression of IL-17 and the number of Th17 cells were reduced in $\mathrm{Tr}^{-/-}$mice compared with WT mice following secondary PPD-bead challenge.

IL- 6 contributes to host resistance against mycobacterial infection due to its immunoenhancing activity (54). Our study showed that specific blockage of IL-6 recapitulates the granuloma phenotype in $\operatorname{Tl} 9^{-/-}$mice with a decreased number of Th17 cells and lower expression of IL-21. IL-21, a cytokine induced by IL-6 in acti- vated T cells, is another cytokine highly expressed by mouse Th17 cells (27). IL-21 potently induces Th17 differentiation and develops a positive feedback loop to further amplify the Th17 response (55). The decreased production of IL-17 in Tlr9-deficient lungs during granuloma formation results from reduced levels of IL-6 and IL-21. In addition, our data demonstrated a decrease in DCs and enhanced macrophage recruitment during pulmonary granuloma formation in Tlr9 $9^{-/-}$mice. The lower production of IL-6 during mycobacterial challenge in Tlr9-deficient DCs may contribute to decreased numbers of Th17 cells during pulmonary granuloma formation. Moreover, we have demonstrated in this study that BCG-pulsed DCs but not macrophages induced IL-17 production by lung $\mathrm{CD}^{+} \mathrm{T}$ cells after restimulation. Denning et al. demonstrated that lamina propria macrophages, which produce IL-10, suppress the differentiation of Th17, while lamina propria DCs induce IL-17 production (36). The data in our study demonstrated enhanced production of IL-10 in BM-derived macrophages during mycobacterial challenge in $\mathrm{Tlr}^{-/-}$mice. The data also suggest these are not alternatively activated macrophages but type II-activated macrophages that produce high levels of IL-10 (56). Because macrophages from $\operatorname{Tl} \mathrm{r}^{-1-}$ mice produce decreased amounts of TNF- $\alpha$ and enhanced levels of IL-10 compared with WT-derived macrophages, they fail to support a Th17 phenotype.

Our results demonstrate that DC-derived Notch ligand dll4 regulates activation of Th17 cytokines in response to Mycobacterium Ags. The present investigation represents what we believe is the first analysis of cell-mediated Th17-related pulmonary granuloma formation in mice and examines the role of dll4 in Th17 cytokine response in both in vivo and in vitro studies. Our studies demonstrate that anti-dll4-treated mice exhibited significantly larger granuloma formation with an accompanied impaired Th17-like response. Our studies also show that dll4 is required for optimal IL-17 production to Ag, but its role in Th17 differentiation is uncertain.

Just as the study of the biology of TLRs had its origin in developmental biology and then transitioned into inflammation and 
A

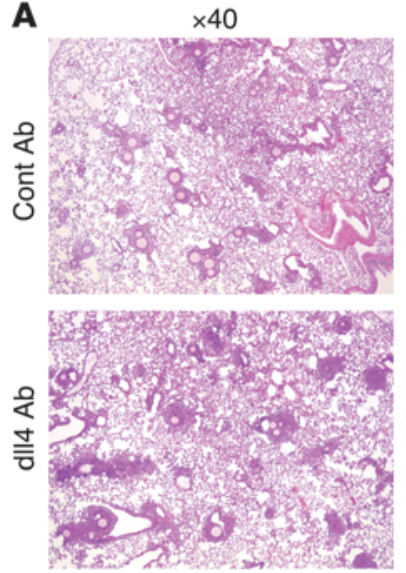

D

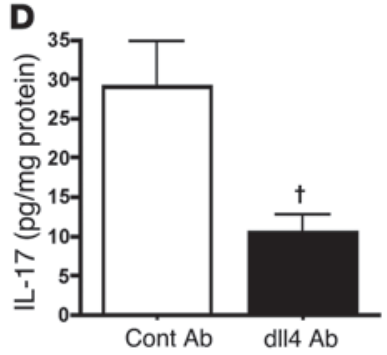

$\times 400$

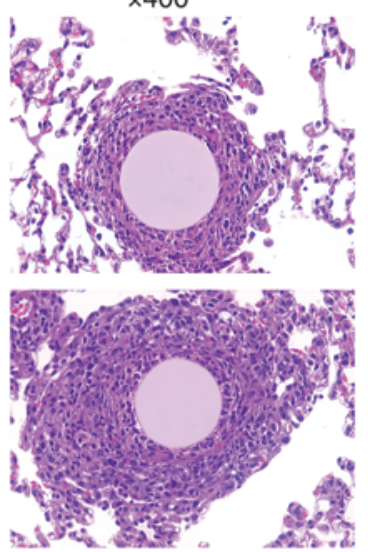

B

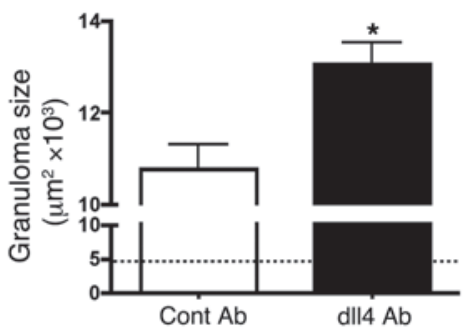

C

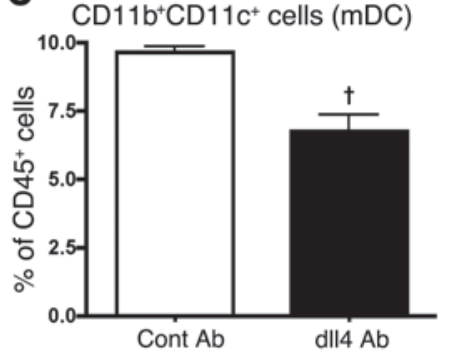

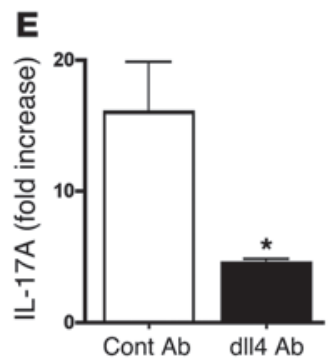
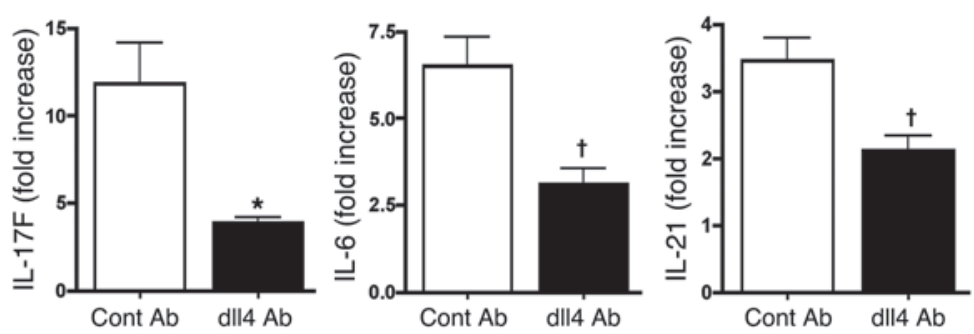

F

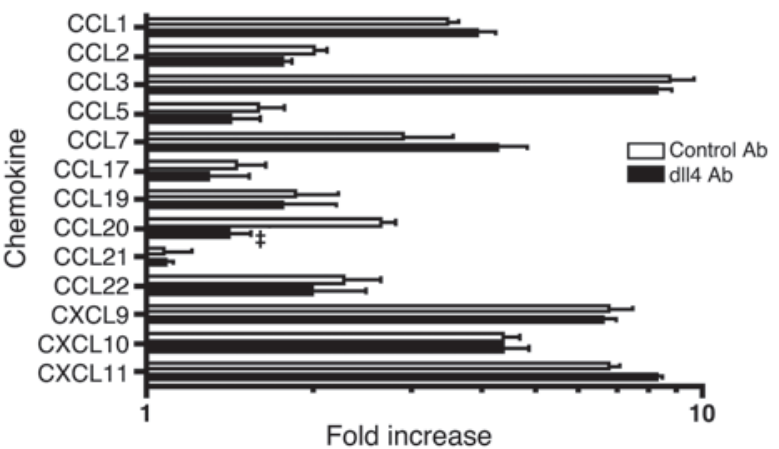

Figure 7

Passive immunization using Abs against dll4 increases granuloma size, abrogates the migration of DCs, and alters the expression of lung Th17-related cytokines and of CCL20. (A) Histological appearance of pulmonary granulomas from WT mice treated with either control Ab or Abs directed against dll 4 at day 4 after granuloma development. H\&E staining. Original magnification, $\times 40 ; \times 400$. Experiments were performed 3 times with similar results. (B) Morphometric analysis of pulmonary granuloma size from mice treated with either control Abs or dll 4 Abs after 4 days of lung granuloma development. ${ }^{*} P<0.01$ compared with control. (C) FACS profiles for mDCs $\left(\right.$ CD11 $\mathrm{b}^{+}$CD11 $\left.\mathrm{C}^{+}\right)$recovered from granulomatous lung cells isolated from day 4 lesions. ${ }^{\dagger} P<0.03$ compared with control. (D) Protein level of IL-17 was measured in whole lungs using a Luminex system. (E) Quantitative real-time PCR was performed on lung mRNA for the cytokines IFN- $\gamma$, IL-4, IL-17A, IL-17F, IL-6, and IL-21. ${ }^{\star} P<0.01$ compared with control. (F) Quantitative real-time PCR was performed on lung mRNA for the indicated chemokines. ${ }^{\ddagger} P<0.02$ compared with control. Data shown are mean \pm SEM from 5 mice and are from a representative experiment of 3 independent experiments. Each time point represents 5 mice per group.

immunity, the field of Notch and Notch ligands began with early discoveries rooted in developmental biology. However, it is now clear that the Notch system is an important bridge between APCs and $\mathrm{T}$ cell communication circuits. DCs can respond to microbial ligands through TLRs (7), which function in the recognition of microbial components and play an important role in both the innate and adaptive immune response. $\mathrm{CD} 4^{+} \mathrm{T}$ cells are essential regulators of adaptive immune responses and inflammatory diseases. Upon antigenic stimulation by $\mathrm{DCs}$, naive $\mathrm{CD}^{+} \mathrm{T}$ cells become activated, expand, and differentiate into various effector $T$ cell help- er subsets termed Th1, Th2 and Th17 and are characterized by the production of distinct cytokines and effector functions (57).

Recent progress shows the importance of Th17 in both innate and acquired immunity against mycobacterial infections (42). However, there has been little research on the role of the NotchNotch ligand system in acquired mycobacterial immunity and on the requirement of Notch activation in the development of Th17 responses. Our data establish that the Notch system is an important bridge between APCs and T cell communication circuits and, further, demonstrate that dll4 neutralization in vivo and in vitro 
A
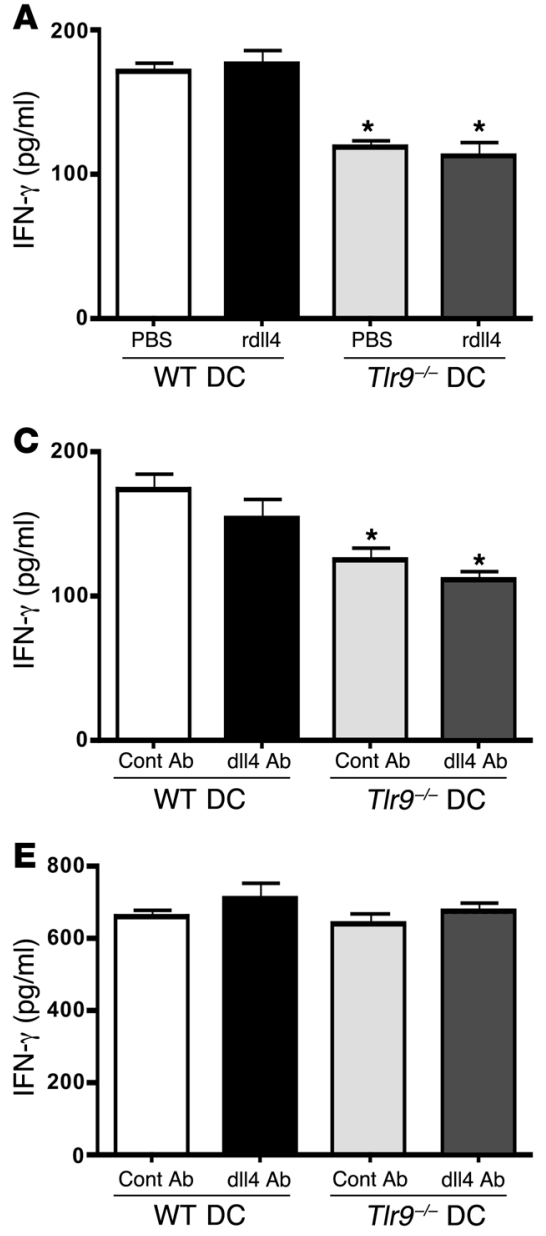

B
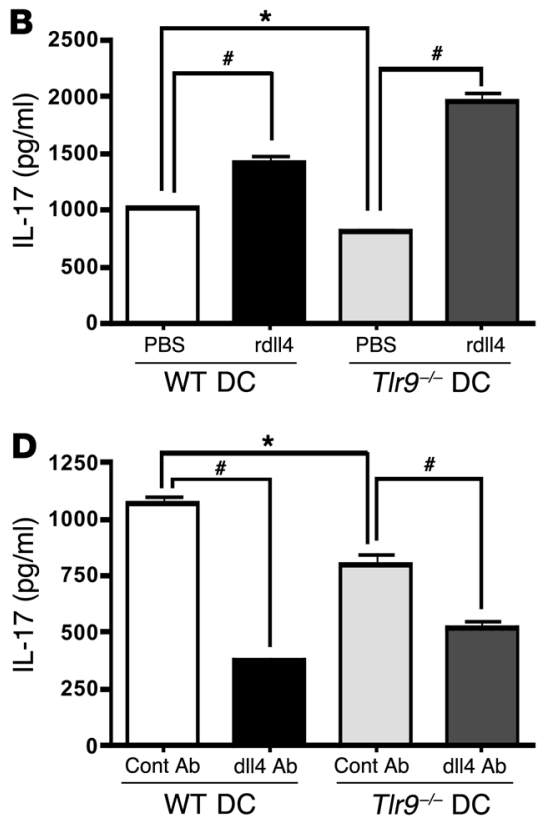

$\mathbf{F}$

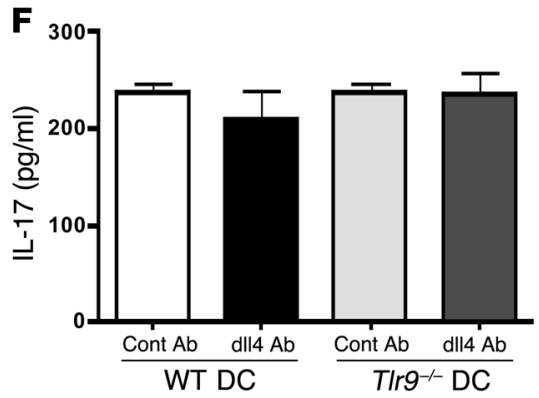

Figure 8

The differentiation of Th17 cells from T cells restimulated with BCG was specifically regulated in a dll4-dependent manner. (A-D) Lung $\mathrm{CD}^{+} \mathrm{T}$ cells were isolated from WT mice and stimulated with BCG-pulsed BM-derived DCs from either WT or T/r9-/- mice. (E and F) Splenic $\mathrm{CD}^{+} \mathrm{T}$ cells were isolated from DO11.10 mice and stimulated with OVA-pulsed BM-derived DCs from either WT or Tlr9-/- mice. Cells were cocultured with recombinant dll4 (rdll4) or PBS controls (A and $\mathbf{B})$ or with anti-dll4 Ab or control IgG (C-F). Supernatants were assayed for IFN- $\gamma$ production $(\mathbf{A}, \mathbf{C}$, and $\mathbf{E})$ or IL-17 production (B, D, and F). ${ }^{*} P<0.05$ compared with WT DC. $\# P<0.01$ compared with PBS (A and $\mathbf{B})$ or control $\mathrm{Ab}(\mathbf{C}$ and $\mathbf{D})$. Data shown are mean \pm SEM and are from a representative experiment of 3 independent experiments. Each time point represents 4 mice per group. during mycobacteria-induced pulmonary granuloma significantly reduces Th17 type cytokine production. Moreover, overexpression of dll4 augmented IL-17 production from T cells isolated from BCG-stimulated lungs. Importantly, dll4 was the primary Notch ligand upregulated on lung DCs and on BM-derived DCs grown in culture during mycobacterial challenge.

dll4 expression from BCG-stimulated DCs from lr $^{-/-}$mice was not totally abrogated, as dll4 was increased 8-fold; however, dll4 expression was increased 40-fold in BCG-stimulated WT DCs. The observation that in the absence of TLR9 some dll4 was still seen suggests that other pathways, potentially other TLRs, may interact with BCG Ags to upregulate dll4, but this upregulation is most potent in the presence of functional TLR9. We further established that these effects were specifically mycobacterial/ Mycobacterium Ag dependent because DCs from WT and Tlr9-1mice induced similar levels of IL-17 production from OVA-specific splenic T cells isolated from DO11.10 mice. Thus, in contrast to BCG, OVA stimulation does not use the TLR9 pathway. These data demonstrate that TLR9 is essential for a positive immune response to mycobacterial challenge and induces the Th17 phenotype via upregulation of dll4. These factors may also contribute to the exacerbation of chronic diseases seen clinically. The molecular mechanism of TLR9-mediated upregulation of dll4 expression during infection remains to be elucidated. It is unknown whether IL-6 or other key cytokines are critical to this process; however, determining the mechanism may reveal a potential clinical target for granuloma diseases.

Chemokines constitute a family of structurally related chemotactic cytokines that direct the migration of leukocytes throughout the body under both physiological and inflammatory conditions (58). CCL20 and CCR6 play a role in the recruitment of immature DCs and their precursors to sites of potential Ag entry $(37,59)$. The lower expression of CCL20 during mycobacterial challenge in both Tlr9-deficient and anti-dll4-treated lung may contribute to the observed decreased DC numbers during pulmonary granuloma formation. In addition, Hirota et al. showed that IL-17-producing Th17 cells express CCR6. Th17 cells induced in vivo in normal mice via homeostatic proliferation similarly express CCR6 as well as CCL20 (59). The above published reports agree with our findings, which show that lower CCL20 expression in both lungs from $\operatorname{Tlr}^{-1-}$ mice and lungs with anti-dll4 $\mathrm{Ab}$ might be correlated with not only impaired DC migration but also reduced numbers of Th17 cells in lungs during mycobacteriainduced pulmonary granuloma formation.

Our findings indicate that dll4 neutralization in vivo during granuloma formation dramatically decreased the Th17 cytokine profile (i.e., IL-17A, IL-17F, IL-6, and IL-21), but had no effect on the Th1 (IFN- $\gamma$ ) or Th2 (IL-4) cytokine profiles (data not shown). Recent studies demonstrate that both bacterial LPS and respiratory virus infection work through TLR4 to induce 


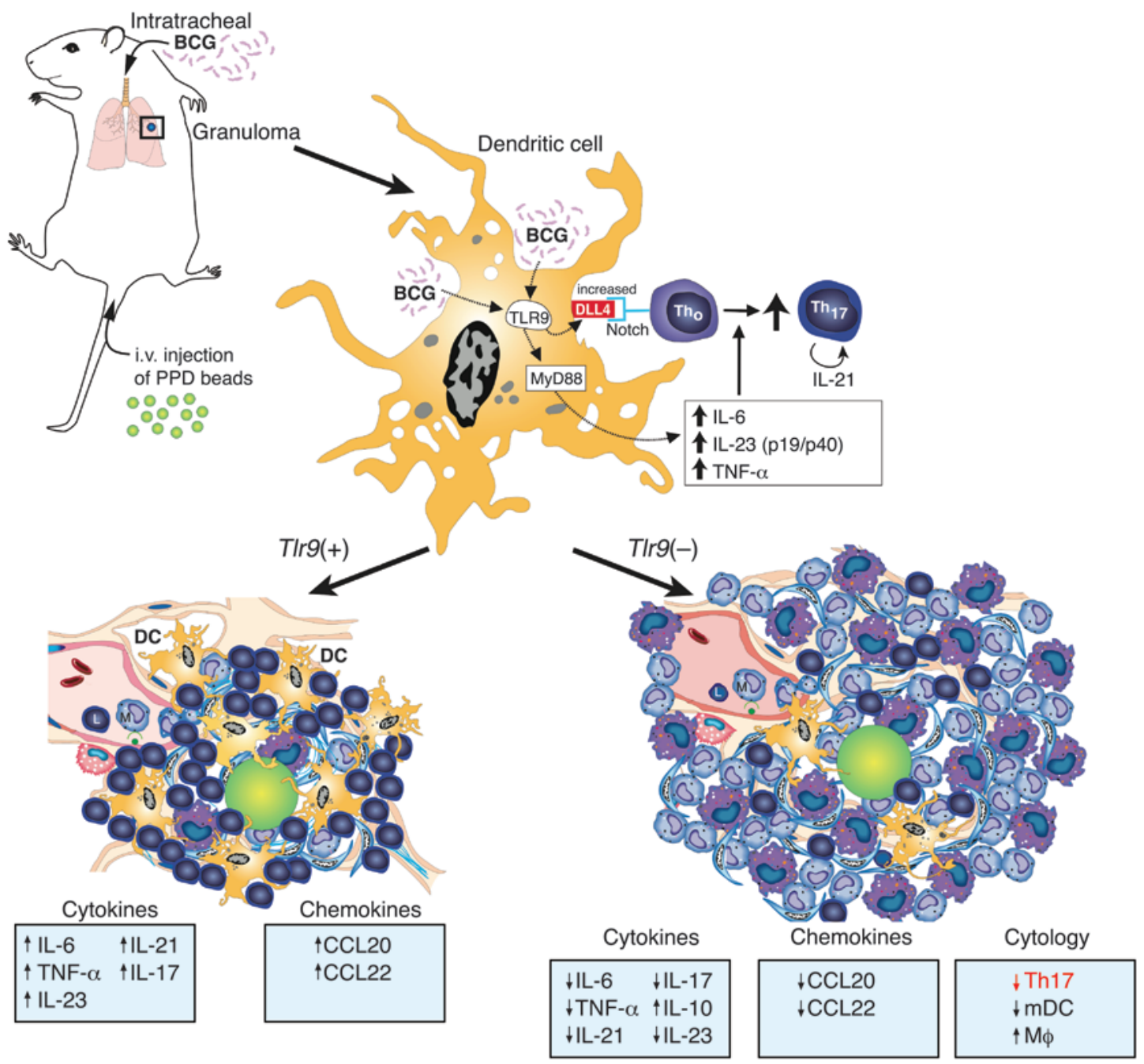

\section{Figure 9}

Schematic representation of the TLR9-Notch ligand (dll4) on Mycobacterium-dependent granuloma formation. DCs play an important role in inducing the differentiation of Th17 cells through the TLR9 effector pathway that upregulates the Notch ligand dll4. In vivo granuloma formation induced by BCG/Mycobacterium Ag demonstrates larger granuloma formation in T/r9-/- mice with decreased numbers of Th17 cells and mDCs in the lungs when compared with lung granulomas from WT mice. Further, TIr9-/- mice showed an increase in IL-10 with a concomitant decrease in Th17 cell-related cytokines (IL-17, IL-6, IL-21, IL-23, and TNF- $\alpha$ ) and a decrease in the levels of the chemokines CCL20 and CCL22, important for DC migration, compared with levels in WT mice. The decreased expression of dll4 and the perturbation of the indicated cytokine and chemokine expression levels led to the abrogation of the Th17 phenotype in the TIr9-/- mice with the concomitant increase in granuloma size. Accompanying these phenomena, there was a decrease in Th17 cells and mDCs in the lungs of TIr9-/- mice and an increase in lung macrophages. L, lymphocyte; $M$, macrophage.

DC-derived dll4 expression, which in turn regulates the balance of Th1/Th2 $(60,61)$. It appears that dll4-regulated immune response of Th17 in mycobacterial infection may be different from other types of infection. Interestingly, both the TLR9 signaling pathway and the robust expression of dll4 on DCs is induced via the MyD88 pathway $(60,61)$. In this study, we demonstrate a decreased expression of dll4 on DCs from Tlr9deficient mice compared with DCs from WT mice, thus linking these pathways together. The present investigation represents an in-depth analysis of Th17 responses with targeted neutralization of dll4 and demonstrates that Th17 cytokine expression was inhibited by neutralizing dll4 and, further, was promoted by dll4 administration.
In summary, we present a comprehensive in vivo analysis of TLR9 participation in a granuloma model induced by mycobacteria-associated Ags. TLR9 deficiency resulted in accelerated granulomatous response and a decreased Th17 cytokine profile during pulmonary granuloma formation. This was associated with a decreased number of $\mathrm{mDCs}$, which can promote the differentiation of Th17 cells, and a significantly increased expression of dll4. Furthermore, Th17 cytokine expression was specifically regulated by dll4 expression on DCs. This study supports the concept that an understanding of dll4 regulation of the Th17 response may provide mechanistic approaches for modifying and controlling the immune response that may ultimately have clinical applicability. 


\section{Methods}

Mice. Male WT mice and male DO11.10 mice, both on a BALB/c background, were purchased from The Jackson ImmunoResearch Laboratories. Male BALB/c mice lacking the TLR9 gene (Tlr9 $\left.9^{-/}\right)$were provided by T.J. Standiford (University of Michigan Medical School, Ann Arbor, Michigan, USA). All mice were used for experiments at 8 to 12 weeks of age. These mice were maintained under specific pathogen-free conditions and provided with food and water ad libitum in the University Laboratory Animal Medicine (ULAM) facility at the University of Michigan Medical School. All animal protocols were approved by ULAM.

Abs. Rat mAbs specific for mouse CD3 (17A2), CD4 (L3T4), CD11b (M1/70), CD11c (HL3), CD16/32 (2.4G2), CD45 (30-F11), CD45R/B220 (RA3-6B2), Gr-1 (RB6-8C5), IFN- $\gamma$ (XMG1.2), IL-4 (11B11), and IL-17 (TC11-18H10) were purchased from BD Biosciences - Pharmingen. Rat anti-F4/80 (CI: A3-1) mAb was purchased from AbD Serotec. Rabbit antimouse dll4 Abs were prepared as previously described (60).

Sensitization and granuloma induction. Mice were sensitized by intratracheal injection of $2.5 \times 10^{5}$ CFUs of BCG (a gift from M. Sandor, University of Wisconsin School of Medicine and Public Health, Madison, Wisconsin, USA) in $100 \mu \mathrm{l}$ of saline. Fourteen days later, mice were challenged i.v. with 5500 Sepharose 4B beads (in $0.5 \mathrm{ml}$ PBS) covalently coupled to PPD, as previously described (5). Mice were sacrificed 4, 8 , or 16 days after i.v. injection. In some experiments, mice were treated with anti-IL-6 (100 $\mu \mathrm{g})$ (eBioscience), anti-dll4, or control Abs on days 0 and 2 of bead challenge.

Histological and immunofluorescent examination. Individual excised lung lobes were inflated and fixed with $10 \%$ buffered formalin for morphometric analysis. The areas of the granulomas were measured in a blinded fashion on H\&E-stained sections of paraffin-embedded lungs using computer-assisted morphometry as previously described (21). For immunofluorescent analysis, lungs were embedded in Tissue-Tek OCT compound (Sakura) and then frozen in liquid nitrogen. Seven-micron cryostat sections were fixed in ice-cold acetone, then incubated with primary Abs followed by appropriate Alexa-labeled secondary Abs (Molecular Probes; Invitrogen). Finally, the sections were analyzed by the Zeiss LSM 510 Confocal Microscope System (Zeiss).

Reverse transcription and real-time quantitative PCR analysis. Total RNA was isolated from whole lungs or cultured cells by using TRIzol (Invitrogen) according to manufacturer's instructions. In brief, a total of $2.0 \mu \mathrm{g}$ of RNA was reverse transcribed to yield cDNA in a $25-\mu 1$ reaction mixture containing $1 \times$ first strand (Life Technologies; Invitrogen), 250 ng oligo (dT) primer, 1.6 $\mathrm{mmol} / \mathrm{l} \mathrm{dNTPs}$ (Invitrogen), $5 \mathrm{U}$ RNase inhibitor (Invitrogen), and $100 \mathrm{U}$ of Moloney murine leukemia virus reverse transcriptase (Invitrogen) at $38^{\circ} \mathrm{C}$ for 60 minutes. The reaction was stopped by incubating the cDNA at $94^{\circ} \mathrm{C}$ for 10 minutes. Real-time quantitative PCR analysis was performed by using the ABI 7700 Sequence Detector System (PE Applied Biosystems). Thermal cycling was performed at $50^{\circ} \mathrm{C}$ for 2 minutes and $95^{\circ} \mathrm{C}$ for 10 minutes, followed by 40 cycles of amplification at $95^{\circ} \mathrm{C}$ for 15 seconds and $55^{\circ} \mathrm{C}$ for 1.5 minutes for denaturing and annealing, respectively.

Protein analysis of cytokine. Murine cytokine levels were measured in $50 \mu \mathrm{l}$ samples from whole-lung homogenates or culture supernatant using a Bio-plex bead-based cytokine assay purchased from Bio-Rad Laboratories. TGF- $\beta$ and IL-23 levels were measured by ELISA as described (eBioscience) (21). The cytokine levels in lung homogenates were normalized to the protein (in milligrams) present in cell-free preparations of each sample measured by the Bradford assay, as described previously (21).

Flow cytometry. Flow cytometric analyses of lung cells were performed as previously described (21). In brief, whole lungs were dispersed in $0.2 \%$ collagenase (Sigma-Aldrich) in RPMI 1640 (MediaTek) and 5\% FBS (Atlas Biologicals) at $37^{\circ} \mathrm{C}$ for 45 minutes to obtain a single-cell suspension. The cells were stained with indicated Abs after 10 minutes of preincubation with CD16/CD32 Abs (Fc block) and fixed overnight with 4\% formalin. For intracellular staining of cytokines, lung cells $\left(1.0 \times 10^{6}\right.$ cells/well $)$ were cultured in 48-well plates containing plate-bound anti-CD3 $(5 \mu \mathrm{g} / \mathrm{ml})$ and soluble anti-CD28 $(2.5 \mu \mathrm{g} / \mathrm{ml})$. After overnight incubation and in the presence of GolgiPlug (BD Biosciences - Pharmingen) for the last 2 hours at $37^{\circ} \mathrm{C}$ and $5 \% \mathrm{CO}_{2}$, the cells were stained for surface markers with FITC-conjugated anti-CD4 Abs, resuspended in fixation/permeabilization solution (BD Cytofix/Cytoperm Kit; BD Biosciences - Pharmingen), and stained with PE-conjugated anti-IFN- $\gamma$, anti-IL-4, or anti-IL-17 Abs respectively. Cells were analyzed using a Cytomics FC 500 (Beckman Coulter), and data were analyzed by FlowJo software (Tree Star Inc.).

Generation of BM-derived DCs and macrophages. BM was harvested from uninfected, normal mice and filtered through nylon mesh. For generation of BM-derived macrophages, BM cells were cultured in L929 cell-conditioned medium as described previously (62). Six days after initial BM culture, BMderived macrophages were transferred to 96 -well plates at a cell density of $4 \times 10^{4}$ cells/well overnight. For generation of BM-derived DCs, BM cells were seeded in T-150 tissue culture flasks at $10^{6}$ cells $/ \mathrm{ml}$ in RPMI $1640-$ based complete medium with GM-CSF $(20 \mathrm{ng} / \mathrm{ml})$ (R\&D Systems) after depletion of erythrocytes with lysis buffer. Six days later, loosely adherent cells were collected and incubated with anti-CD11c coupled to magnetic beads for isolation of conventional DCs from the GM-CSF cultures (Miltenyi Biotec). BM-derived DCs were purified using positive selection for the specific cells by running the cell suspension through a magnetic column. The purity of CD11c was more than $95 \%$, as determined by flow cytometry. The cells were plated on 96-well plates at a cell density of $3 \times 10^{5}$ cells/well overnight. The next day, macrophages and DCs were stimulated with BCG (MOI $=0,0.1$, or 1.0$)$ or CPG $(1 \mu \mathrm{M})$ (Cell Sciences). Six hours later, RNA was isolated. Twenty-four hours later, cell-free supernatants were removed for analysis in a Bio-Plex bead-based cytokine assay. In some experiments, the cells were plated on 96-well plates at a cell density of $4 \times 10^{4}$ cells/well overnight. The next day, macrophages and DCs were infected with BCG $(\mathrm{MOI}=1.0)$ for 2 hours, then washed before addition of $\mathrm{T}$ cells.

In vitro $T$ cell treatments. Fourteen days after intratracheal injection with $2.5 \times 10^{5} \mathrm{CFU}$ s of $\mathrm{BCG}, \mathrm{CD} 4^{+} \mathrm{T}$ cells from lungs were isolated with a magnetic bead column (Miltenyi Biotec). More than $97 \%$ of cells were CD4 positive. These cells $\left(2 \times 10^{5}\right.$ cells/well $)$ were exposed to BCG-pulsed BMderived DCs or macrophages on 96-well plates at an $\mathrm{APC} / \mathrm{CD} 4^{+}$cell ratio of 1:5. In some experiments, splenic $\mathrm{CD} 4^{+} \mathrm{T}$ cells from DO11.10 mice were isolated with a magnetic bead column (>97\% purity). These cells $\left(2 \times 10^{5}\right.$ cells/well) were exposed to OVA-pulsed BM-derived DCs on 96-well plates at a $\mathrm{DC} / \mathrm{CD}^{+}$cell ratio of $1: 10$. Supernatants were harvested 48 hours later for cytokine protein analysis.

Statistics. Two-tailed Student's $t$ test was used to compare groups. Values of $P<0.05$ were considered significant.

\section{Acknowledgments}

This work was supported by NIH grants P01-HL031963, HL092845, HL074024, and HL031237. We thank Robin Kunkel, Valerie Stolberg, Pam Lincoln, and Holly Evanoff for their technical assistance as well as Judith Connett for her critical reading of the manuscript.

Received for publication March 17, 2008, and accepted in revised form November 5, 2008.

Address correspondence to: Steven L. Kunkel, Immunology Program, Department of Pathology, University of Michigan Medical School, 4071 BSRB, 109 Zina Pitcher Place, Ann Arbor, Michigan 48109-2200, USA. Phone: (734) 936-1020; Fax: (734) 764-2397; E-mail: slkunkel@umich.edu. 
1. Boros, D.L. 1978. Granulomatous inflammations. Prog. Allergy. 24:183-267.

2. El-Zammar, O.A., and Katzenstein, A.L. 2007. Pathological diagnosis of granulomatous lung disease: a review. Histopathology. 50:289-310.

3. Dye, C., et al. 2007. Global tuberculosis control: surveillance, planning, financing. WHO Report 2007. C. Dye, K. Floyd, M. Hosseini, A. Piatek, and C. Watt, editors. WHO. Geneva, Switzerland. 277 pp.

4. Chensue, S.W., Ruth, J.H., Warmington, K., Lincoln, P., and Kunkel, S.L. 1995. In vivo regulation of macrophage IL-12 production during type 1 and type 2 cytokine-mediated granuloma formation. J. Immunol. 155:3546-3551.

5. Chensue, S.W., et al. 1994. Cytokine responses during mycobacterial and schistosomal antigeninduced pulmonary granuloma formation. Production of Th1 and Th 2 cytokines and relative contribution of tumor necrosis factor. Am. J. Pathol. 145:1105-1113.

6. Chensue, S.W., Warmington, K.S., Ruth, J.H., Lincoln, P., and Kunkel, S.L. 1995. Cytokine function during mycobacterial and schistosomal antigeninduced pulmonary granuloma formation. Local and regional participation of IFN-gamma, IL-10, and TNF. J. Immunol. 154:5969-5976.

7. Akira, S., Uematsu, S., and Takeuchi, O. 2006 Pathogen recognition and innate immunity. Cell. 124:783-801.

8. Akira, S. 2003. Mammalian Toll-like receptors. Curr. Opin. Immunol. 15:5-11.

9. Trinchieri, G., and Sher, A. 2007. Cooperation of Toll-like receptor signals in innate immune defence. Nat. Rev. Immunol. 7:179-190.

10. Uematsu, S., and Akira, S. 2007. Toll-like receptors and type I interferons. J. Biol. Chem. 282:15319-15323.

11. Jo, E.K., Yang, C.S., Choi, C.H., and Harding, C.V. 2007. Intracellular signalling cascades regulating innate immune responses to Mycobacteria: branching out from Toll-like receptors. Cell Microbiol. 9:1087-1098

12. Krutzik, S.R., and Modlin, R.L. 2004. The role of Toll-like receptors in combating mycobacteria. Semin. Immunol. 16:35-41.

13. Ryffel, B., et al. 2005. Innate immunity to mycobacterial infection in mice: critical role for toll-like receptors. Tuberculosis. 85:395-405.

14. Abel, B., et al. 2002. Toll-like receptor 4 expression is required to control chronic Mycobacterium tuberculosis infection in mice. J. Immunol. 169:3155-3162.

15. Bafica, A., et al. 2005. TLR9 regulates Th1 responses and cooperates with TLR2 in mediating optimal resistance to Mycobacterium tuberculosis. J. Exp. Med. 202:1715-1724.

16. Sugawara, I., et al. 2003. Mycobacterial infection in TLR2 and TLR6 knockout mice. Microbiol. Immunol. 47:327-336.

17. Hemmi, H., et al. 2000. A Toll-like receptor recognizes bacterial DNA. Nature. 408:740-745.

18. Jakob, T., Walker, P.S., Krieg, A.M., Udey, M.C., and Vogel, J.C. 1998. Activation of cutaneous dendritic cells by CpG-containing oligodeoxynucleotides: a role for dendritic cells in the augmentation of Th1 responses by immunostimulatory DNA. J. Immunol. 161:3042-3049.

19. Sparwasser, T., et al. 1998. Bacterial DNA and immunostimulatory $\mathrm{CpG}$ oligonucleotides trigger maturation and activation of murine dendritic cells. Eur. J. Immunol. 28:2045-2054.

20. Krieg, A.M., et al. 1995. CpG motifs in bacterial DNA trigger direct B-cell activation. Nature. 374:546-549.

21. Ito, T., et al. 2007. TLR9 activation is a key event for the maintenance of a mycobacterial antigenelicited pulmonary granulomatous response. Eur. J. Immunol. 37:2847-2855.
22. Stockinger, B., and Veldhoen, M. 2007. Differentiation and function of Th17 T cells. Curr. Opin. Immu nol. 19:281-286.

23. Harrington, L.E., et al. 2005. Interleukin 17-producing $\mathrm{CD}^{+}$effector $\mathrm{T}$ cells develop via a lineage distinct from the $\mathrm{T}$ helper type 1 and 2 lineages. Nat. Immunol. 6:1123-1132.

24. Weaver, C.T., Harrington, L.E., Mangan, P.R., Gavrieli, M., and Murphy, K.M. 2006. Th17: an effector $\mathrm{CD} 4 \mathrm{~T}$ cell lineage with regulatory $\mathrm{T}$ cell ties. Immu nity. 24:677-688.

25. Mangan, P.R., et al. 2006. Transforming growth factor-beta induces development of the $\mathrm{T}(\mathrm{H}) 17$ lineage. Nature. 441:231-234.

26. Bi, Y., Liu, G., and Yang, R. 2007. Th17 cell induction and immune regulatory effects. J. Cell. Physiol. 211:273-278.

27. Nurieva, R., et al. 2007. Essential autocrine regulation by IL- 21 in the generation of inflammatory T cells. Nature. 448:480-483.

28. Brombacher, F., Kastelein, R.A., and Alber, G. 2003. Novel IL-12 family members shed light on the orchestration of Th1 responses. Trends Immunol. 24:207-212.

29. Veldhoen, M., Hocking, R.J., Atkins, C.J., Locksley, R.M., and Stockinger, B. 2006. TGF $\beta$ in the context of an inflammatory cytokine milieu supports de novo differentiation of IL-17-producing T cells. Immunity. 24:179-189.

30. Osborne, B.A., and Minter, L.M. 2007. Notch signalling during peripheral T-cell activation and differentiation. Nat. Rev. Immunol. 7:64-75.

31. Amsen, D., et al. 2004. Instruction of distinct CD4 $\mathrm{T}$ helper cell fates by different notch ligands on antigen-presenting cells. Cell. 117:515-526.

32. Dallman, M.J., Smith, E., Benson, R.A., and Lamb, J.R. 2005. Notch: control of lymphocyte differentiation in the periphery. Curr. Opin. Immunol. 17:259-266.

33. Ansel, K.M., Djuretic, I., Tanasa, B., and Rao, A. 2006. Regulation of Th2 differentiation and Il4 locus accessibility. Annu. Rev. Immunol. 24:607-656.

34. Raymond, T., et al. 2007. Toll-like receptors, notch ligands, and cytokines drive the chronicity of lung inflammation. Proc. Am. Thorac. Soc. 4:635-641.

35. Komiyama, Y., et al. 2006. IL-17 plays an important role in the development of experimental autoimmune encephalomyelitis. J. Immunol. 177:566-573.

36. Denning, T.L., Wang, Y.C., Patel, S.R., Williams, I.R., and Pulendran, B. 2007. Lamina propria macrophages and dendritic cells differentially induce regulatory and interleukin 17-producing $\mathrm{T}$ cell responses. Nat. Immunol. 8:1086-1094.

37. Schutyser, E., Struyf, S., and Van Damme, J. 2003. The CC chemokine CCL20 and its receptor CCR6. Cytokine Growth Factor Rev. 14:409-426.

38. Yamashita, U., and Kuroda, E. 2002. Regulation of macrophage-derived chemokine (MDC, CCL22) production. Crit. Rev. Immunol. 22:105-114.

39. Tokunaga, T., et al. 1984. Antitumor activity of deoxyribonucleic acid fraction from Mycobacterium bovis BCG. I. Isolation, physicochemical charcterization, and antitumor activity. J. Natl. Cancer Inst. 72:955-962.

40. von Meyenn, F., et al. 2006. Toll-like receptor 9 contributes to recognition of Mycobacterium bovis Bacillus Calmette-Guerin by Flt3-ligand generated dendritic cells. Immunobiology. 211:557-565.

41. Cooper, A.M., et al. 2002. Mice lacking bioactive IL-12 can generate protective, antigen-specific cellular responses to mycobacterial infection only if the IL-12 p40 subunit is present. J. Immunol. 168:1322-1327.

42. Matsuzaki, G., and Umemura, M. 2007. Interleukin-17 as an effector molecule of innate and acquired immunity against infections. Microbiol. Immunol. 51:1139-1147.

43. Oppmann, B., et al. 2000. Novel p19 protein engages
IL-12p40 to form a cytokine, IL-23, with biological activities similar as well as distinct from IL-12. Immunity. 13:715-725.

44. Umemura, M., et al. 2007. IL-17-mediated regulation of innate and acquired immune response against pulmonary Mycobacterium bovis bacille CalmetteGuerin infection. J. Immunol. 178:3786-3796.

45. Cua, D.J., et al. 2003. Interleukin-23 rather than interleukin-12 is the critical cytokine for autoimmune inflammation of the brain. Nature. 421:744-748.

46. Murphy, C.A., et al. 2003. Divergent pro- and antiinflammatory roles for IL-23 and IL-12 in joint autoimmune inflammation. J. Exp. Med. 198:1951-1957.

47. Kolls, J.K., and Linden, A. 2004. Interleukin-17 family members and inflammation. Immunity. 21:467-476.

48. Rutitzky, L.I., Lopes de Rosa, J.R., and Stadecker, M.J. 2005. Severe CD4 T cell-mediated immunopathology in murine schistosomiasis is dependent on IL-12p40 and correlates with high levels of IL-17. J. Immunol. 175:3920-3926.

49. Aggarwal, S., Ghilardi, N., Xie, M.H., de Sauvage, F.J., and Gurney, A.L. 2003. Interleukin-23 promotes a distinct CD4 $\mathrm{T}$ cell activation state characterized by the production of interleukin-17. J. Biol. Chem. 278:1910-1914.

50. Khader, S.A., et al. 2005. IL-23 compensates for the absence of IL-12p70 and is essential for the IL-17response during tuberculosis but is dispensable for protection and antigenspecific IFN $-\gamma$ response if IL-12 p70 is available. J. Immunol. 175:788-795.

51. Goldsack, L., and Kirman, J.R. 2007. Half-truths and selective memory: Interferon gamma, CD4 (+) $\mathrm{T}$ cells and protective memory against tuberculosis. Tuberculosis. 87:465-473.

52. Khader, S.A., et al. 2007. IL-23 and IL-17 in the establishment of protective pulmonary $\mathrm{CD}^{+} \mathrm{T}$ cell responses after vaccination and during Mycobacterium tuberculosis challenge. Nat. Immunol. 8:369-377

53. Wozniak, T.M., Ryan, A.A., Triccas, J.A., and Britton, W.J. 2006. Plasmid interleukin-23 (IL-23), but not plasmid IL-27, enhances the protective efficacy of a DNA vaccine against Mycobacterium tuberculosis infection. Infect. Immun. 74:557-565.

54. Label, C.H., et al. 1997. Lethal tuberculosis in Interleukin-6-deficient mutant mice. Infect. Immun. 65:4843-4849.

55. Korn, T., et al. 2007. IL-21 initiates an alternative pathway to induce proinflammatory $\mathrm{T}(\mathrm{H}) 17$ cells. Nature. 448:484-487.

56. Edwards, J.P., Zhang, X., Frauwirth, K.A., and Mosser, D.M. 2006. Biochemical and functional characterization of three activated macrophage populations. J. Lenkoc. Biol. 80:1298-1307.

57. Bettelli, E., et al. 2006. Reciprocal developmental pathways for the generation of pathogenic effector Th17 and regulatory T cells. Nature. 441:235-238.

58. Yoshie, O., Imai, T., and Nomiyama, H. 2001. Chemokines in immunity. Adv. Immunol. 78:57-110.

59. Hirota, K., et al. 2007. Preferential recruitment of CCR6-expressing Th17 cells to inflamed joints via CCL20 in rheumatoid arthritis and its animal model. J. Exp. Med. 204:2803-2812.

60. Schaller, M.A., et al. 2007. Notch ligand Delta-like 4 regulates disease pathogenesis during respiratory viral infections by modulating Th 2 cytokines. J. Exp. Med. 204:2925-2934.

61. Skokos, D., and Nussenzweig, M.C. 2007. CD8 DCs induce IL-12-independent Th1 differentiation through Delta 4 Notch-like ligand in response to bacterial LPS. J. Exp. Med. 204:1525-1531.

62. Joshi, A.D., et al. 2008. A systemic granulomatous response to Schistosoma mansoni eggs alters responsiveness of bone marrow-derived macrophages to Toll-like receptor agonists. J. Leukoc. Biol. 83:314-324. 